\title{
A Novel Design Framework for Generation and Parametric Modification of Yacht Hull Surfaces
}

\author{
Shahroz Khan*,1 Erkan Gunpinar ${ }^{1}$ Kemal Mert Dogan ${ }^{1}$ \\ ${ }^{1}$ Istanbul Technical University
}

\begin{abstract}
This paper proposes a new design framework for the parametric design and shape modification of a yacht hull. In this framework, the hull is divided into three regions (entrance, middle and run) and each region is represented separately. In this way, a designer has better design flexibility so that higher design variations of the hull can be achieved. Each region consists of keel line(s), deck line, chine line(s) and station lines that are represented using Bezier curves and these lines are called feature curves. A 3D surface model of a yacht hull is obtained by generating Coons patches using feature curves. Shape operators are also introduced and implemented for the modification of the given hull shape while considering some quality criteria such as hull fairness. Experiments in this study show that a variety of hull shapes can be generated using the proposed design framework with the application of the shape operators.
\end{abstract}

Keywords: Computer Aided Design, Parametric Design, Yacht Hulls, Surface Modeling, Bezier Curves.

\section{Introduction}

In computer aided design, parametric design techniques provide sophisticated platforms for designers in order to perform the design process efficiently. Using parametric design techniques, the design process starts by parameterizing the important features of design models, which are called design parameters. The relationships between design parameters are then determined, which are called design constraints. Initializing an appropriate number of design parameters and constraints is a crucial step of parametrization.

There are some proposed techniques in the literature [1]-[22] that are intended to describe the parametric modeling of a yacht hull, but most of these are neither sophisticated nor flexible enough to produce variant hull forms. Calkins et al. [1] and Pérez-Arribas [2] proposed techniques for parametric generation of yacht hulls, but these techniques are less flexible in terms of design variations. This is because the hull form is formed by defining only three feature curves: sheer line (deck line), center line (keel line) and chine line(s). Pérez-Arribas [2] uses these feature curves to create lofted surfaces in order to obtain the 3D surface model of a hull. Therefore, these fewer number of curves can restrict the ability of the design framework in [1] and [2] to generate hulls with different geometries. Furthermore, these techniques can only produce particular types of planing hulls.

The design framework presented in this paper can produce a variety of shapes of displacement and semi-displacement hulls besides the planing hulls. By using this framework, designers can create hulls from classical to modern yachts with different chine line(s) and bow shapes. All types of yacht hulls that are shown in Figure 1 (c) can be produced using the proposed design framework, whereas the techniques proposed in [1] and [2] cannot produce all of these

*Email: shahrozkhan2020@gmail.com, khansh@itu.edu.tr Adress: Istanbul Technical University, School of Mechanical Engineering, Inonu Caddesi, No: 65, Gumussuyu, 34437, Istanbul, TURKEY, Tel: +90-212-2931300 Fax: +90-212-2450795 


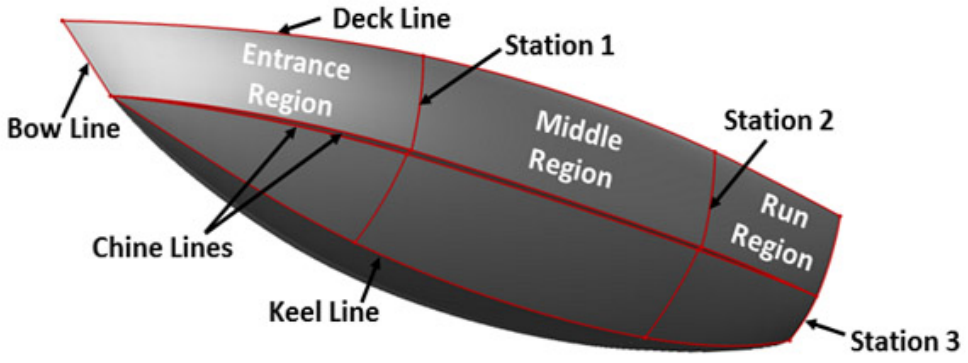

(a)

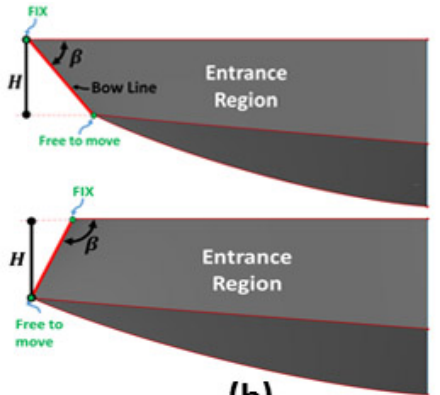

(b)
Planing Hulls

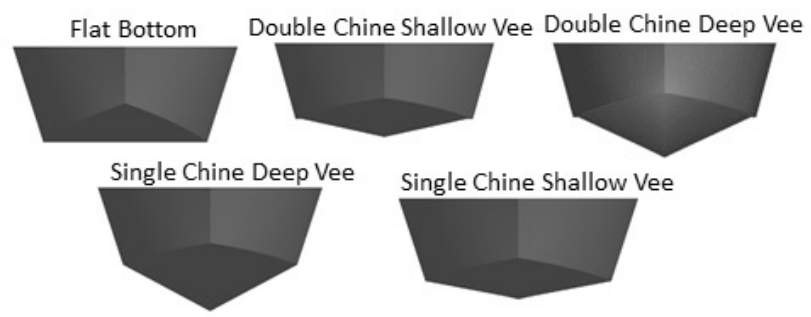

Semi-displacement hull
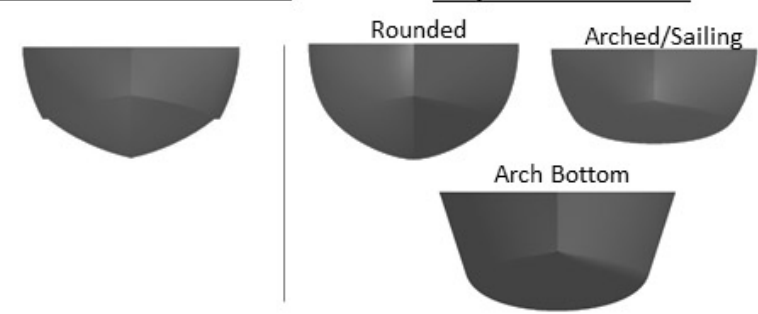

(c)

Figure 1. (a) Arrangement of feature curves in the proposed framework that are used to form the overall shape of the yacht hull. (b) Modification of the bow angle $\beta$ with the vertical length $H$ of bow line. During the modification of $\beta$, the value of $H$ should be maintained. (c) Most common types of displacement and planing yacht hulls.

hull types. The technique in [1] cannot generate any of the displacement and semi-displacement yacht hulls and the technique in [2] cannot produce flat bottom planing yacht hull and displacement yacht hulls. However, there are other techniques in the literature $[3,4]$ which can generate displacement yacht hulls, but not any type of planing yacht hulls. Table 1 provides the comparison between the capability of existing techniques and the proposed method. As a result, to the best of our knowledge, there is lack of more robust techniques in the literature which can ensure the parametric generation of both planing and displacement yacht hulls at the same time.

In the proposed design framework, hull is divided into three regions: entrance, middle and run regions as shown in Figure 1 (a). Such a division can give the designers a design flexibility so that the hulls with variant geometries can be generated. The shape of each hull region is defined by deck line, keel line(s), chine line(s) and station lines which are considered as feature curves. The arrangement of the feature curves can be seen in Figure 1 (a). Design parameters/constraints are defined on/between the feature curves. Figure 1 (b) illustrates two design parameters: the vertical length of the bow line $H$ and the angle of the bow line $\beta$ with the horizontal axis. A design constraint is also shown that fixes the upper point of the bow line during the modification of $\beta$. Shape operators are developed as well allowing modification of each design parameter while satisfying the design constraints. Using these operators, design variations of a given hull can be obtained. These shape operators change the hull shape while taking the following quality criteria into account:

1. Geometric Continuity: $G^{0}$ and $G^{1}$ continuities must be maintained at the connection point of two adjacent feature curves. Otherwise, surfaces generated from these curves will not be connected tightly and smoothly at the connection points.

2. Hull Fairness: Fairness of feature curves should be preserved after applying shape operators so that uneven design modification(s) of the hull form can be avoided.

3. Independent Modification of Parameters: Shape operators are designed based on design parameters. While changing the value of a single parameter, values of other parameters should remain the same as much as possible. In this way, modification of any parameter will not result in the change of other parameter values (see Figure 1 (b)). This criterion provides better modification in terms of variant hull form generations. 
In order to develop a new design, designers start with an empty design or they retrieve an existing design from database which is then modified to obtain the desired design. The later approach is commonly preferred by designers and thus utilized in many industries. In this work, it is assumed that a yacht hull is given as input that is obtained using the proposed design framework which will be modified to obtain the desired hull shape using shape operators while taking predefined design constraints into account.

\section{Related Work}

During the last decade, several advancements have been made to the field of parametric generation of ship and yacht hulls. Many researchers have proposed various geometric techniques for creating different types of parametric hull forms. Calkins et al. [1] proposed a method to define the planing hull form in the conceptual design stage of the design process. This method utilizes a parametric technique to define stations as straight lines in order to create the overall shape of the hull. However, straight line stations restrict the ability of this method to create different types of planing hulls. Recently, Pérez-Arribas [2] suggested a parametric technique for the creation of hard-chine planing hulls. In this method, the hull geometry is created by decomposing the surface geometry of the hull into curves. Constraints and design parameters are defined on these curves for hull design variations. 3D curves are produced by fitting B-spline curves between 3D data points on 2D orthogonal views of the hull. A drawback of this method is that it does not utilize any fairness criterion during the formulation of curves and requires the 2D orthogonal information of the hull form. Furthermore, in terms of producing variant hull types, these techniques [1] and [2] are less flexible because of the reason mentioned earlier in Sec. 1.

Mancuso [3] introduced a method for the parametric design of a sailing yacht in which hull form is defined by using B-spline surfaces. An optimization technique is then used to fair these surfaces by considering the defined parameters and constraints. A simple parametric design approach to generate sailing hulls and round bilge hulls was proposed by Pérez et al. [4]. This method creates hull lines on the basis of the user defined hydrostatic coefficients and then generates B-spline surfaces on these lines for 3D hull models. A parametric method to create complex ship hull forms was proposed by Kim and Nowacki [5] in which multiple B-spline surfaces are used to create a hull shape. Mathematical operators such as Boolean operator and surface blending are then used to create connections between these surfaces. Zalek et al. [6] proposed a technique for the transformation of a feasible parent hull form into a new hull form. In this technique, hull undergoes different phases of linear and nonlinear transformation to achieve desired hull form.

Nam and Parsons [7] proposed a method to create initial hull form by dividing the overall shape into five regions: stem, entrance, midbody, run and stern. In this method, control points of NURBS surfaces are modified to alter the overall shape of the hull. A design-oriented parametric technique was introduced by Abt et al. [8] to generate ship hull forms. In this technique, design variations in the hull form are achieved by considering some selected parameters as constraints, and other parameters are changed automatically by using a mathematical formulation. Pérez and Suárez [9] developed ship hulls by using quasi-developable B-spline surface. In this method hull curves such as keel line, chines and sheer lines are created by using B-spline curves, and then quasi-developable B-spline surfaces are created on these feature curves. Harries et al. [10] and Hochkirch et al. [11] have investigated the optimal design of sailing yachts from the geometrical and the hydrodynamic point of view.

Rodríguez and Fernández-Jambrina [12] proposed a CAD based technique called programed design in order to define and parameterize the hull form. This method uses three different parameterization methodologies (global parameterization, geometric parameterization and parameterization by transformations) to create ship hulls. Kuiper [13] and Keane [14] worked on developing the parametric ship hull forms using the conformal mapping techniques. Keane [14] also observed the impact of the defined parameter on the stability of the hull. In another study, PérezArribas et al. [15] developed a method for automatically creating hull forms by utilizing a fairing algorithm for NURBS surfaces, and then used these surfaces to create the hull form. Zhang et al. [16] used a parametric design approach for rapid creation of ship hulls in order to optimize the hull's hydrodynamics. A geometric parametric technique to produce complex planing tunnel vessels was proposed by Ghassabzadeh and Ghassemi [17]. Parsons [18], Lowe and Steel [19] and Papanikolaou [20] also studied this topic by using advanced optimization techniques like genetic algorithms. Jacqin et al. [21] also developed a hull form optimization method using a free surface RANSE solver. 
There is a considerable amount of research done by many researchers in this field, but most of these researchers have focused on the parametric design of large container ship hulls. It was observed by the authors of this paper that there is a lack of a single parametric approach that can work for the creation of different types of yacht hulls at the same time such as displacement, planing and semi-displacement hulls. This unavailability motivated the authors to work on this topic, and the proposed design framework is a more generalized and innovative design framework for yacht hull designers. Using this design framework, a yacht hull can be modified with less effort and in a shorter amount of time.

Table 1. Comparison table for related works.

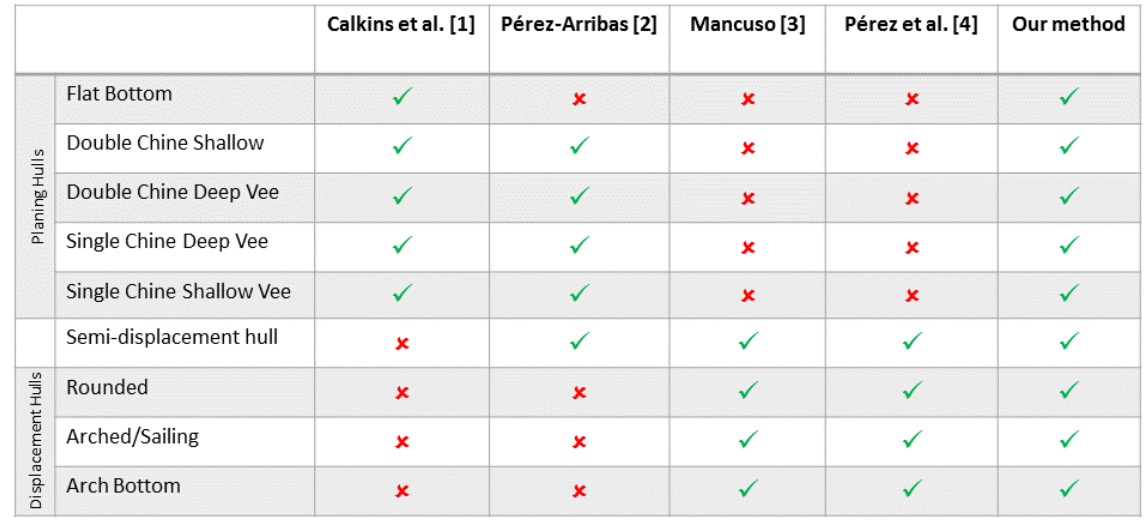

\section{Design Framework}

The proposed design schema can help to create an extensive range of hull forms for different types of yachts such as motor, sailing, expedition and open yachts. Designers can create different types of displacement hulls (arched, rounded), planing hulls (deep vee, shallow vee, flat bottom) and semi-displacement hulls. A yacht hull is divided into three regions: entrance, middle and run regions as shown in Figure 1 (a). Each region consists of independent feature curves representing characteristic lines of the hull such as deck line, keel line(s), chine line(s) and station lines as shown in Figure 2 (a). The entrance region has an extra feature curve for the bow. Each station consists of keel-thickness line, chine-thickness line and two sub-station lines. Feature curves form the overall shape of the hull and are represented by using Bezier curves. Bezier curves are utilized in the proposed design framework as they have compact and intuitive mathematical description so that they are easy to manipulate. Several works in the literature have also used these curves in hull form modeling [1,22]. Mathematically, a Bezier curve can be represented by Equation 1.

$$
P(u)=\sum_{i=0}^{n} P_{i} B_{i, n}(u) \quad ; \quad u \in[0,1]
$$

where $P_{i}$ represents the control points and $B_{i, n}(u)$ is the corresponding basis function called Bernstein Polynomial which is defined as: $B_{i, n}(u)=C(n, i) u^{i}(1-u)^{n-i}$, and $C(n, i)=n ! / i !(n-i) !$. The cubic Bezier curve with four control points $(n+1=4)$ can be defined as in Equation 2 .

$$
P(u)=P_{0}(1-u)^{3}+P_{1} 3 u(1-u)^{2}+P_{2} 3 u^{2}(1-u)^{2}+P_{3} u^{3}
$$

In this work, cubic Bezier curves are utilized to represent feature curves except for the bow, chine-thickness and keel-thickness lines of which linear curves (i.e., lines) are used instead. Using cubic and linear Bezier curves, flexible hull design modification can be achieved by changing positions of the control points of the feature curves. The technical details of each region are as follows: 
- Entrance Region (ER): The feature curves of this region are deck line, keel line(s), chine line(s), bow line and station-1. Station-1 consists of two sub-station lines (upper and lower line of station-1)), a keel-thickness line and chine-thickness line (see Figure 2 (a)). The chine-thickness line enables the generation of double chine yacht hulls. If there is a chine thickness, two chine lines are formed: the inner chine line and the outer chine line. In the presence of keel thickness, there is one more keel line (called offset keel line), which is offset to the main keel line in the positive $X$ direction. The keel-thickness line is defined to create flat bottom yacht hulls. Chine-thickness line is specified between the last control point of the upper line of station-1 $\left(P_{S 3}^{e}\right)$ and the first control point of the lower line of station-1 $\left(P_{S 4}^{e}\right)$. Keel-thickness line is established between the last control point of the lower line of station-1 $\left(P_{S 7}^{e}\right)$ and the last control point of the keel line $\left(P_{K 3}^{e}\right)$.

- Middle Region (MR): The feature curves of the middle region are deck line, keel line(s), chine line(s) and station-2. Station-2 also consists of sub-station lines (above and below the chine line(s)), chine-thickness line and keel-thickness line as shown in Figure 2 (a). The feature curves of the entrance and middle region are connected at station-1. The amid-ship is located at the middle region, which mostly provides the reference to define the maximum beam and maximum depth of hulls.

- Run Region (RR):The feature curves of the run region are deck line, keel line(s), chine line(s) and station-3 as shown in Figure 2 (a). Station-3 consists of sub-station lines, keel-thickness line and chine-thickness line. Station-3 defines the transom of the hull, which plays an important role in hull design with respect to hydrostatic performance. Station-2 serves as a connection line between the middle and run regions.

\subsection{Generation of Surfaces}

A 3D surface model of a hull is generated by interpolating Coons patches between feature curves separately in each region. For better aesthetics, $G^{0}$ and $G^{1}$ geometric continuities are maintained between the connection points of feature curves (at station-1 and station-2). Otherwise, the generated Coons patches will not be smooth at these connection points. In each region, there are at least four Coons patches, if a double chine and keel thickness exists. The entrance region consists of one rectangular and three triangular Coons patches. The first rectangular patch is created between the deck line, the outer chine line, the bow line and the upper line of station- 1 . The second Coons patch is a triangular patch which is created using the inner chine, outer chine and chine-thickness lines. The third Coons patch is generated between the inner chine line, the lower line of station- 1 and the offset keel line. Finally, the fourth Coons patch is generated between the keel line, the keel-thickness line and the offset keel line (see Figure 2 (c)). Coons patches for the middle and run regions are obtained in a similar fashion. To obtain the right body portion of the hull, these Coons patches are mirrored along the $Y Z$ plane.

\subsection{Defining Design Parameters}

Design parameters that are meaningful in the hull design process are listed in Table 2 and can be seen in Figure 2 (b). Each region has its own design parameter set. $L_{e}, L_{m}$ and $L_{r}$ define the length of entrance, middle and run regions, respectively. These parameters provide a benefit to designers for modifying the length of each region separately. In order to achieve a hydrodynamic hull form, the designer can alter the length of the entrance region $L_{e}$ without affecting the rest of the hull shape. The width (beam) of entrance, middle and run regions are defined by $B_{e}, B_{m}$ and $B_{r}$ respectively, and the depth of each region is defined by $D_{e}, D_{m}$ and $D_{r}$. The parameter $F_{b}$ is the vertical height of the foremost point of chine line(s) on the bow. $F_{e}, F_{m}$ and $F_{r}$ are the vertical positions of chine at station-1, 2 and 3 respectively, and the draft of the hull can be altered by utilizing these parameters. The parameters $C_{e}, C_{m}$ and $C_{r}$ specify the thickness (distance) that is between the inner and outer chine lines for double chine hulls.

The parameter $R_{e k}$ is the minimum radius of curvature for the keel line(s) in the entrance region which is employed to alter the forefoot radius of the hull. During the creation of a plumb bow, one can reduce the forefoot radius by altering the $R_{e k}$. The parameters $R_{e s 1}$ and $R_{e s 2}$ specify the minimum radius of curvature for the upper and lower lines of station-1. During the creation of rounded or arch displacement hulls, $R_{e s 2}$ can be modified to make the hull more rounded below the chine line(s). For similar purposes, the minimum radius of curvature for the upper and lower lines of station- 2 is denoted by $R_{m s 1}$ and $R_{m s 2}$ and that of station-3 is denoted by $R_{r s 1}$ and $R_{r s 2}$. The vertical height of the bow is denoted by $H$ and keel thickness is denoted by $K . K$ produces a flat panel at the keel of the hull. The parameter 

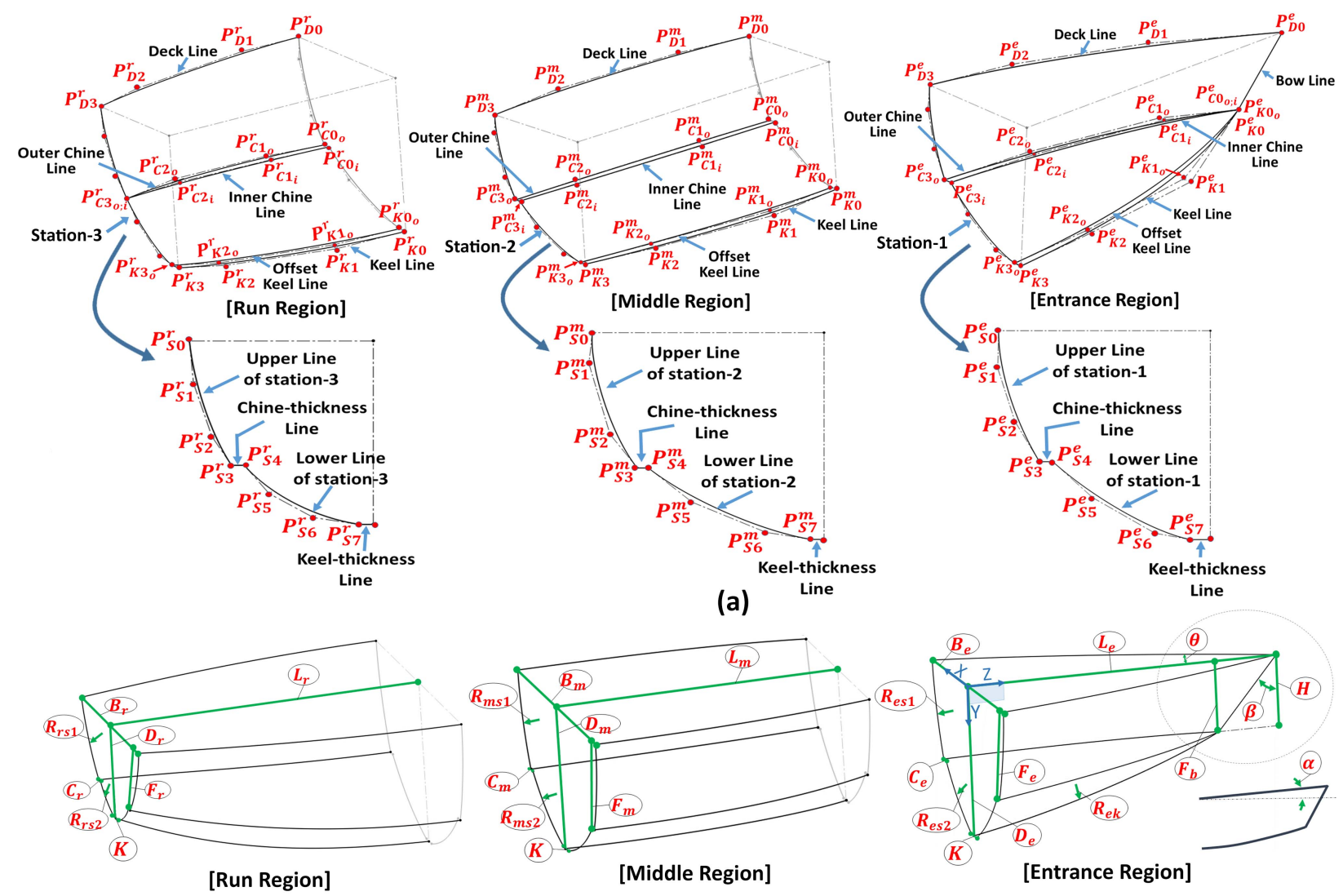

(b)
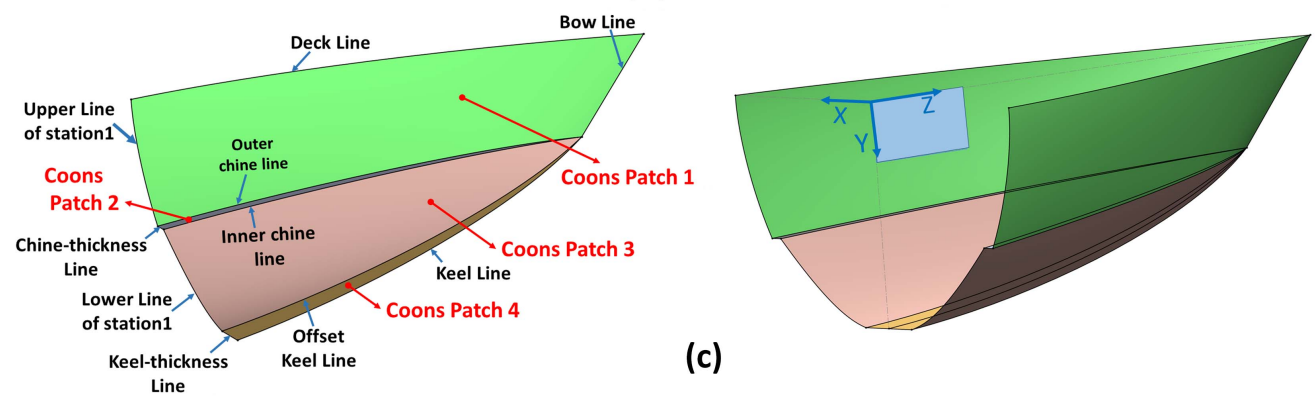

(c)

Figure 2. (a) Entrance, middle and run regions with particular arrangement of feature curves and their control points. (b) Design parameters defined on the feature curves of entrance, middle and run regions. (c) Generation of Coons patches using feature curves for the entrance region.

$\beta$ represents the angle of the bow, while $\theta$ represents the entrance angle which is positioned between the Z-Y plane and the foremost end tangent of the deck line in the entrance region. The parameter $\theta$ has significant contribution to the hull's hydrodynamics. $\alpha$ is the sheer angle which is employed between the deck plane (the plane on which deck line is created) and Z-X plane. This parameter creates sheer forward on the hull.

\subsection{Generating Surface Model of the Initial Yacht Hull}

We assume that the designer has a surface model of an initial yacht hull which can be generated in the way described in this section. The 3D network of feature curves for the initial model is created by utilizing the 2D orthogonal views of a yacht hull. 2D orthogonal details of a model are generally used as the initial drafts by the designers. Top and side views of the hull are partitioned into three regions and feature lines for each region are 
Table 2. List of design parameters defined on the entrance, middle and run regions of the hull.

\begin{tabular}{|l|l|l|l|}
\hline Name & Description & Name & Description \\
\hline$L_{e}$ & Length of ER & $C_{m}$ & Chine thickness at station-2 \\
\hline$L_{m}$ & Length of MR & $C_{r}$ & Chine thickness at station-3 \\
\hline$L_{r}$ & Length of RR & $R_{e k}$ & Minimum radius of curvature for keel line(s) in ER \\
\hline$B_{e}$ & Width(Beam) of ER & $R_{e s 1}$ & Minimum radius of curvature for upper line of station-1 \\
\hline$B_{m}$ & Width(Beam) of MR & $R_{e s 2}$ & Minimum radius of curvature for lower line of station-1 \\
\hline$B_{r}$ & Width(Beam) of RR & $R_{m s 1}$ & Minimum radius of curvature for upper line of station-2 \\
\hline$D_{e}$ & Depth of ER & $R_{m s 2}$ & Minimum radius of curvature for lower line of station-2 \\
\hline$D_{m}$ & Depth of MR & $R_{r s 1}$ & Minimum radius of curvature for upper line of station-3 \\
\hline$D_{r}$ & Depth of RR & $R_{r s 2}$ & Minimum radius of curvature for lower line of station-3 \\
\hline$F_{e}$ & Vertical chine position at station-1 & $H$ & Vertical length of bow line \\
\hline$F_{m}$ & Vertical chine position at station-2 & $K$ & Keel thickness \\
\hline$F_{r}$ & Vertical chine position at station-3 & $\beta$ & Bow angle \\
\hline$F_{b}$ & Vertical chine position at bow & $\alpha$ & Sheer angle \\
\hline$C_{e}$ & Chine thickness at station-1 & $\theta$ & Entrance angle \\
\hline
\end{tabular}

created (see Figure 3). In order to create a bow line and keel line, the side view is utilized and three cubic Bezier curves are plotted for the keel line and a linear line is plotted for the bow. Similarly, the deck line is plotted using the top view. Station lines of each region are created using the front view.
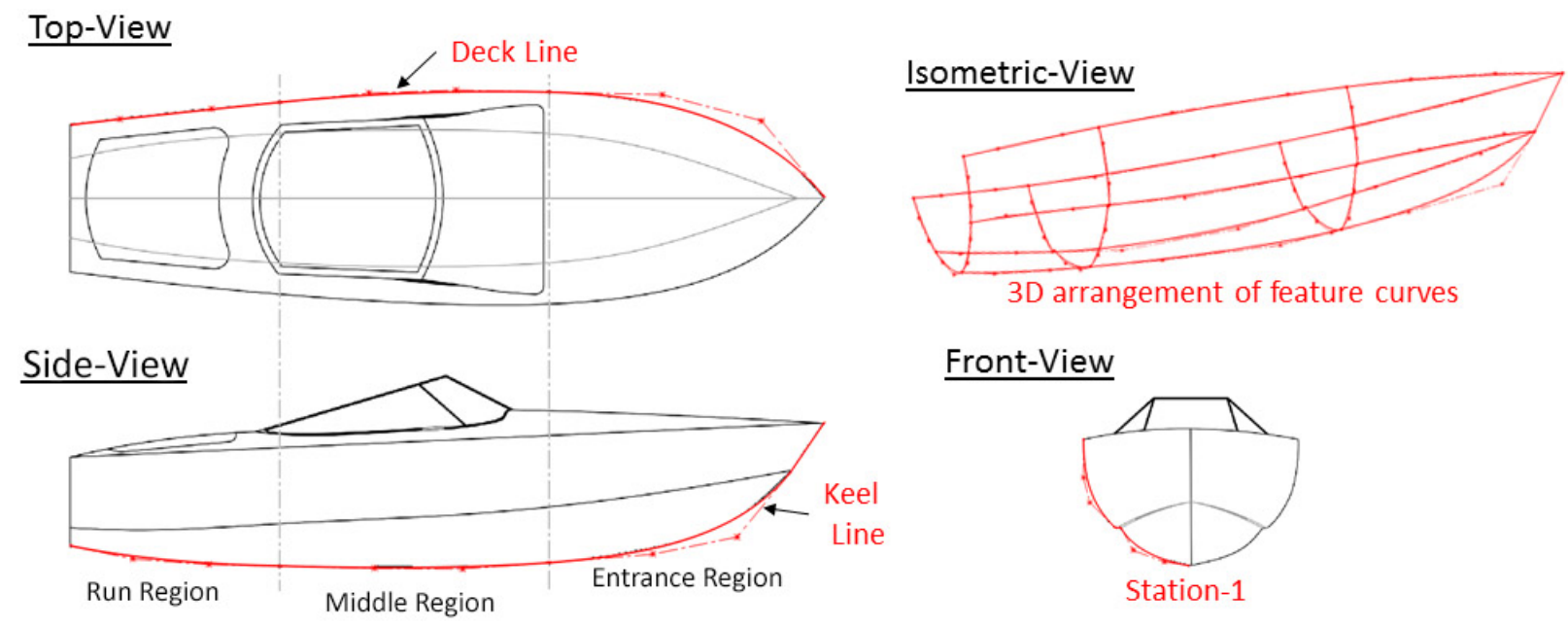

Front-View

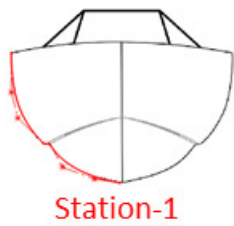

Figure 3. Generating surface model of the initial hull.

\section{Shape Operators}

Shape Operators modify the hull shape while considering the quality criteria mentioned in Sec. 1. We classify these operators (see Table 3) into two groups, Group-1 and Group-2, on the basis of the techniques used for their implementation. Shape Operators in Group-1 are based on an iterative approach and control points of the feature curves are determined while maximizing the value of the objective function that will be introduced in Sec. 4.2. Specific techniques are proposed for the shape operators in Group-2. 
Table 3. List of defined shape operators.

\begin{tabular}{|l|l|l|l|l|}
\hline \multicolumn{5}{|c|}{ Shape Operators } \\
\hline Elongation & Bow Angle & Chine Remover & Keel Thickness & Mroup-2 \\
\hline Widening & Entrance Angle & Chine Inserter & Bow Vertical Length & Station Concavity \\
\hline Deepening & Sheer Angle & Chine Thickness & Chine Position & \\
\hline
\end{tabular}

\subsection{Quality Criteria for Hull Shape Modification}

In computer aided design, geometric continuities should be achieved in order to have smooth transitions at the connections of neighboring curves or surfaces. To achieve $G^{0}$ continuity between two cubic Bezier curves, the end point of one curve must be coincident with the start point of its neighboring curve. To maintain smoothness between two neighboring curves, at least $G^{1}$ continuity should be present, which requires that the tangents of the two curves be the same at their connection points. In other words, the last polygon segment of the first curve and the first polygon segment of the second curve should be collinear (see Figure 4 (a)).
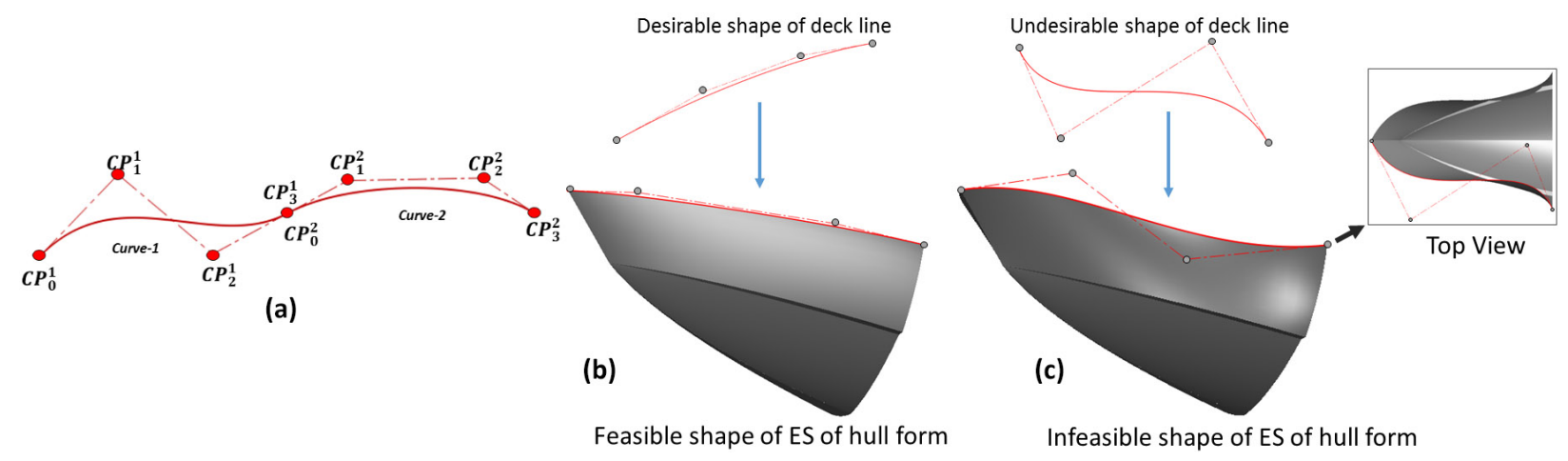

Figure 4. (a) $G^{0}$ and $G^{1}$ continuity is maintained between the connection point of Curve-1 and Curve-2. (b) Desirable shape of deck line forming a feasible shape of the entrance region of the hull form. (c) Undesirable shape of deck line forming an infeasible shape of the entrance region of the hull form.

Fairness of the hull is an important design requirement in terms of hull aesthetics and hydrodynamic performance [23]. Unfair hull surfaces indicate the presence of isolated bumps. Occurrence of these bumps in the surfaces would affect the hydrodynamic behavior of hull form by increasing its resistance $[15,4]$. Fairness is an odd term, which has no explicit mathematical definition and usually changes from one application to another. According to Farin [24, 25, 26], Sariöz [27], Salvi and Várady [28], Kuragano and Kasono [29, 30], and Kuragano and Yamaguchi [31] a curve can be considered fair if the curvature plot of the curve changes smoothly along its parametric length. During modification, it is preferable to obtain a curve whose curvature distribution is similar to that of the input curve. In this way, the input and modified curves can have similar shapes, and curves with undesired shapes can be avoided. Utilizing undesired feature curves results in the generation of infeasible hull surface shapes. For example, the entrance region of hull with a shape as that in Figure 4 (c) is uncommon and can be considered an infeasible hull. By approximating curvature plots of the input and modified curves, the modified curve can be fair if the input curve is fair. Kuragano and Kasono's technique $[29,30]$ is utilized to compare curvature distributions of the input and modified curve, and evaluates the similarity between these two curves. In this work, a given curve is modified by comparing its curvature plot with that of the input curve. 


\subsection{Implementation of Shape Operators in Group-1}

For fair and feasible modification of the feature curves, an Iterative Approach (IA) is utilized and an objective function $(F)$ is introduced based on the proposed technique in $[29,30]$ :

$$
F=\frac{R_{I} \cdot R_{M}}{\left|R_{I}\right|\left|R_{M}\right|}
$$

Curvature of a cubic Bezier curve can be computed using Equation 4:

$$
\kappa(u)=\frac{\dot{P}(u) \times \ddot{P}(u)}{|\dot{P}(u)|^{3}}
$$

$\dot{P}(u)$ and $\ddot{P}(u)$ denote the first and second order derivatives of Equation 2, respectively. $\kappa(u)$ is the curvature at a parametric length $(u)$. The radius of curvature $R(u)$ is the reciprocal of $\kappa(u)$ and is expressed as $R(u)=\frac{1}{\kappa(u)}$. The $i^{\text {th }}$ radius of curvature distribution of a curve along its parametric length is denoted by $R\left(u_{i}\right)$ where $i=1,2,3, \cdots, \xi$ and $u_{i}$ is a monotonically increasing parametric length. $\xi$ denotes the total number of curve divisions for computing the radius of curvature distribution. The terms $R_{I}$ and $R_{M}$ in the objective function $F$ are the $\xi$ dimensional vectors containing the discrete values of the radius of curvature for the input and modified feature curves, respectively (see Equation 5).

$$
R_{I}=\left[\begin{array}{c}
R_{I}\left(u_{1}\right) \\
R_{I}\left(u_{2}\right) \\
\vdots \\
R_{I}\left(u_{\xi}\right)
\end{array}\right] ; \quad R_{M}=\left[\begin{array}{c}
R_{M}\left(u_{1}\right) \\
R_{M}\left(u_{2}\right) \\
\vdots \\
R_{M}\left(u_{\xi}\right)
\end{array}\right]
$$

Positions of inner control points for the modified curve are determined while maximizing the value of the objective function $F$. The objective function $F$ ranges from 0 to 1 and the value of $F$ is close to 1 for similar curves. The iterative approach taken in this work is illustrated using a modification example in Figure 5. Let $L$ is the length of a curve and is increased from $L$ to $L^{\prime}$ while keeping tangents at the start and end points of the curves (ang1 and ang2) constant. The ang 2 is kept fixed, so that the connecting polygon segments remain collinear in order to maintain $G^{1}$ continuity between the two connecting feature curves of two different regions, and ang 1 is kept fixed in order to keep other design parameters unaffected (remember the criterion 3 in Sec. 1). The control point $P_{3}$ is fixed and $P_{0}$ is moved along the $Z$ by the amount of $L^{\prime}-L$. Candidate positions of inner control points $\left(\dot{P}_{1}\right.$ and $\left.\dot{P}_{2}\right)$ of the modified curve are computed as follows: $\dot{P}_{1}=P_{0}+\hat{\mathrm{n}}_{1} t$ and $\dot{P}_{2}=P_{3}+\hat{\mathrm{n}}_{2} k, \hat{\mathrm{n}}_{1}$ and $\hat{\mathrm{n}}_{2}$ are the unit vectors from $P_{0}$ to $P_{1}$ and from $P_{3}$ to $P_{2}$, respectively (see Figure 5 (a)). The above equations represent two line segments in the $\hat{\mathrm{n}}_{1}$ and $\hat{\mathrm{n}}_{2}$ directions with start points $P_{0}$ and $P_{3}$, respectively. $t$ and $k$ denote the parametric lengths for these segments. At $t=0$ and $k=0$ the inner control points $P_{1}$ and $P_{2}$ are $P_{0}$ and $P_{3}$, respectively.
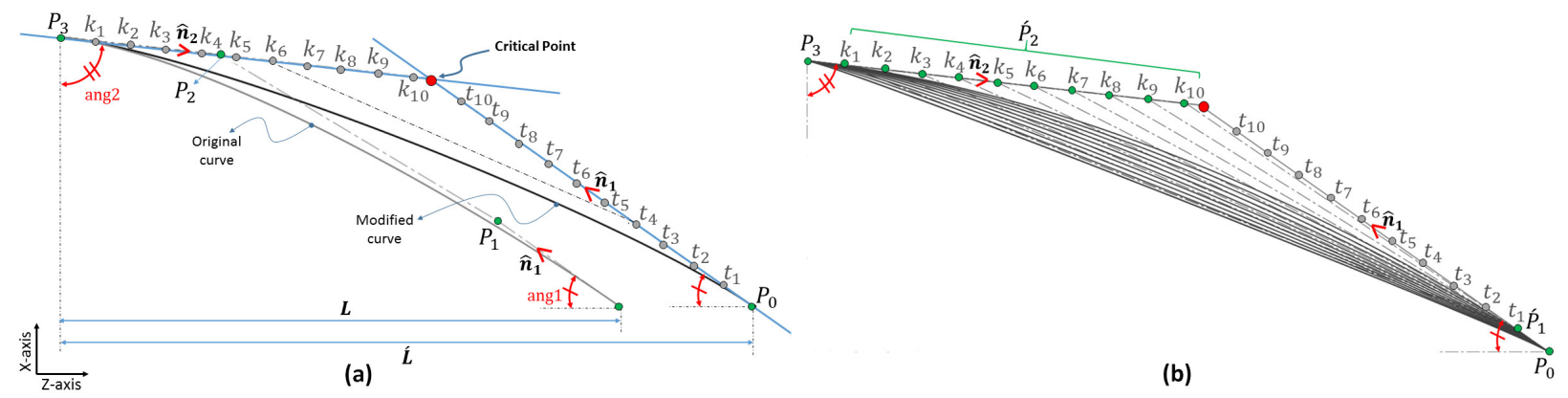

Figure 5. (a) Modified curve with parametric lengths of candidate positions for its inner control points $\dot{P}_{1}$ and $\dot{P}_{2}$ (b) First ten iterations of the IA is illustrated. $t_{1}$ is the parametric length of first candidate position for $\dot{P}_{1}$ and parametric lengths for $\dot{P}_{2}$ change iteratively from $k_{1}$ to $k_{10} . F$ value is computed in each iteration. 
In 2D Euclidean space, for $\hat{\mathrm{n}}_{1} \neq \hat{\mathrm{n}}_{2}$ two line segments intersect at some parametric position $\hat{t}$ and $\hat{k}$, and this intersection point is considered as a critical point. The inner control points cannot exceed the critical point, otherwise the curve will have self-intersection. The overall problem for this modification example can be formulated as:

$$
\begin{aligned}
& P_{1}^{\prime}=P_{0}+\hat{\mathrm{n}}_{1} t \\
& P_{2}=P_{3}+\hat{\mathrm{n}}_{2} k
\end{aligned}
$$

Find $\boldsymbol{t}$ and $\boldsymbol{k}$ which maximizes $F$

subject to constraints

$$
\begin{aligned}
& 0 \leq t \leq \hat{t} \\
& 0 \leq k \leq \hat{k}
\end{aligned}
$$

0 and $\dot{t}$ are lower and upper bounds of $t$, while 0 and $k$ are the lower and upper bounds of $k$. Candidate positions for inner control points are computed by dividing line segments from the start to the critical point into $\mu$ number of points. $\mu$ is called a division constant. After the division process, $t_{1}, t_{2}, t_{3}, \cdots, t_{\mu}$ denote the parametric lengths of candidate positions for $\dot{P}_{1}$. And $k_{1}, k_{2}, k_{3}, \cdots, k_{\mu}$ denote the parametric lengths of candidate positions for $\dot{P}_{2}$. By setting larger values for $\mu$, more candidate positions can be generated so that many solutions for $\dot{P}_{1}$ and $\dot{P}_{2}$ can be searched. Thus a solution nearer to the optimum one can be found at the expense of computation time. Figure 5 (b) illustrates 10 candidate positions for the $\mu=10$ setting: $t_{1}, t_{2}, t_{3}, \cdots, t_{\mu}$ for $P_{1}$, and $k_{1}, k_{2}, k_{3}, \cdots, k_{10}$ for $P_{2} .10 \times 10$ number of iterations will be performed and the value of function $F$ will be obtained from all candidate positions of $\dot{P}_{1}$ and $\dot{P}_{2}$. Finally, the candidate positions which maximizes the value of $F$ will be the new positions of inner control points $\dot{P}_{1}$ and $\dot{P}_{2}$. There are curves whose generated line segments have no intersection (i.e., $\hat{n_{1}}$ equals to $\hat{n_{2}}$ ). Therefore, no critical point can be computed. However, curves without intersection points are not used in yacht hull modeling according to our observations.

\subsubsection{Elongation Shape Operator}

Elongation shape operator is developed to modify the length parameters $L_{e}, L_{m}$ and $L_{r}$ defined on each region. The shape of the deck line, chine line(s) and keel line(s) of hull form is affected by this shape operator. Control point positions of the deck line, chine line(s) and keel line(s) in the entrance region (see Figure 6 (a)) are obtained while taking the following approach into account, which is summarized in the pseudo-code below:

1: The control points $P_{D 3}^{e}, P_{K 3}^{e}$ and $P_{C 3_{i, o}}^{e}$ are fixed (If double chine exists then in expression $P_{C 3_{i, o}}^{e}, P_{C 3_{i}}^{e}$ represents the last control point of inner chine line and $P_{C 3_{o}}^{e}$ represents the last control point of outer chine in the entrance region. Similarly, chine line control points of other regions are represented).

2: Move $P_{D 0}^{e}, P_{K 0}^{e}$ and $P_{C 0_{i, o}}^{e}$ by $\pm \Delta L_{e}$ (change of amount in $L_{e}$ ) in $\pm Z$ direction while keeping $F_{b}, H$ and $\beta$ constant (remember the criterion 3 in Sec. 1).

3: Compute positions of inner control points $\dot{P}_{D 1}^{e}, \dot{P}_{D 2}^{e}, \dot{P}_{K 1}^{e}, \dot{P}_{K 2}^{e}, \dot{P}_{C 1_{i, o}}^{e}$ and $\dot{P}_{C 2_{i, o}}^{e}$ using IA in Sec. 4.2.

A similar approach is taken to change lengths of the middle and run regions. During the change in $L_{m}$, control points of all feature curves in the entrance region are also moved by $\pm \Delta L_{m}$ in the $\pm Z$ direction. Similarly, control point positions of both middle and entrance regions are moved by $\pm \Delta L_{r}$ in the $\pm Z$ direction to modify the length $L_{r}$ of the run region.

\subsubsection{Widening Shape Operator}

The widening shape operator modifies the width or beam $\left(B_{e}, B_{m}\right.$ and $\left.B_{r}\right)$ of the hull regions (see Figure 2 (a, b)). Station-1 is situated between the entrance and middle regions. Therefore, control point positions of deck line and chine line(s) in both entrance and middle regions are modified in order to maintain $G^{1}$ continuities at the connection points (see Figure $6(\mathrm{~b})$ ). The below pseudo-code below summarized the shape operator for the $B_{e}$ parameter:

1: The control points $P_{D 0}^{e}, P_{C 0_{i, 0}}^{e}, P_{D 3}^{m}, P_{C 3_{i, o}}^{m}$ and $P_{S 7}^{e}$ are fixed. 


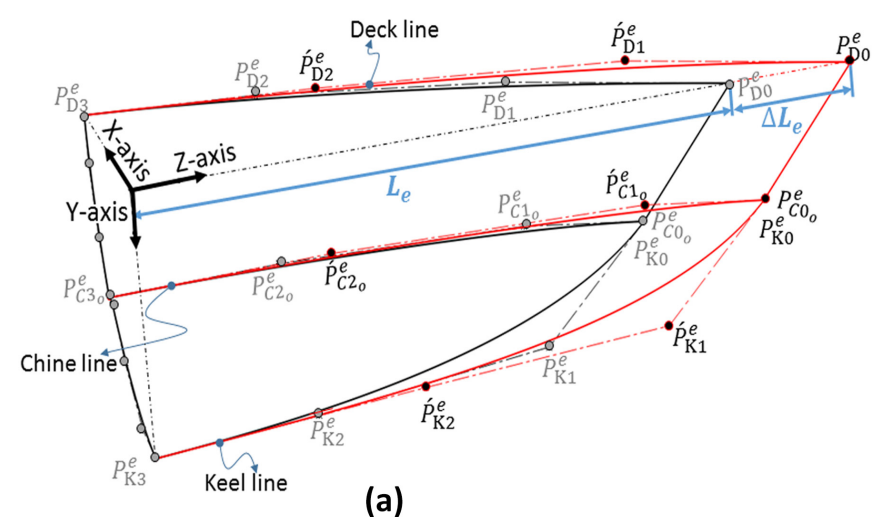

(a)

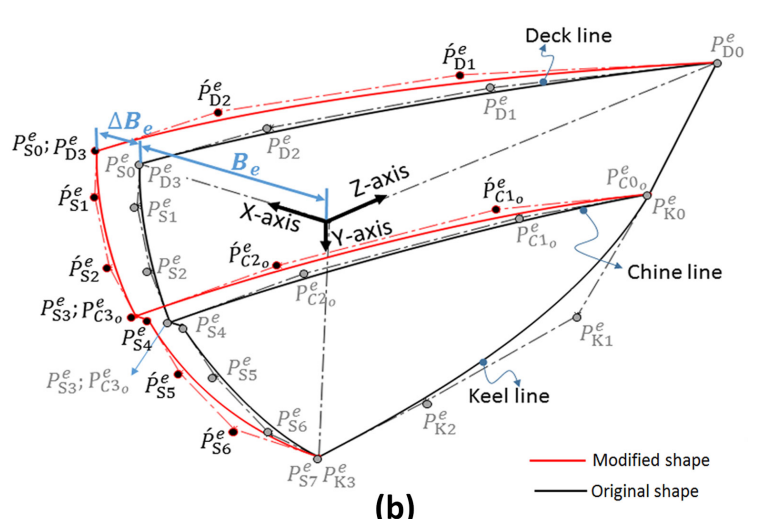

(b)

Figure 6. (a) Implementation of elongation shape operator on the entrance region. (b) Implementation of widening shape operator on the entrance region.

2: Move $P_{D 3}^{e}, P_{D 0}^{m}, P_{S 0}^{e}, P_{C 3_{i: o}}^{e}, P_{C 0_{i, o}}^{m}, P_{S 3}^{e}$ and $P_{S 4}^{e}$ in the $\pm X$ direction by $\pm \Delta B_{e}$ ( change in $B_{e}$ ), (Notice that $C_{e}$ is kept constant).

3: Compute positions of inner control points $\dot{P}_{D 1}^{e}, \dot{P}_{D 2}^{e}, \dot{P}_{C 1_{i: o}}^{e}, \dot{P}_{C 2_{i: o}}^{e}, \dot{P}_{D 1}^{m}, \dot{P}_{D 2}^{m}, \dot{P}_{C 1_{i: o}}^{m}, \dot{P}_{C 2_{i: o}}^{m}, \dot{P}_{S 1}^{e}, \dot{P}_{S 2}^{e}, \dot{P}_{S 5}^{e}$ and $\dot{P}_{S 6}^{e}$ using IA.

The parameter $B_{m}$ is modified in a similar manner and its modification affects control point positions of the deck line and chine line(s) in both middle and run regions. For the modification of $B_{r}$, only the shape of the deck line and chine line(s) in the run region, and upper and lower lines of station-3 are affected.

\subsubsection{Deepening Shape Operator}

The approach taken for deepening shape operator is similar to that of the widening shape operator. Its application on station-1 changes the depth $D_{e}$ and modifies control point positions of the keel line(s) in the entrance and middle regions along with the lower line of station-1. However, the upper line of station-1 is not modified in order to keep the parameter $F_{e}$ constant (see Figure $2(\mathrm{a}, \mathrm{b})$ ). The pseudo-code below summarizes the approach used to implement this shape operator for $D_{e}$.

1: The control points $P_{K 0}^{e}, P_{K 3}^{m}$ and $P_{S 4}^{e}$ are fixed.

2: Move $P_{K 3}^{e}, P_{K 0}^{m}, P_{S 7}^{e}$ by $\pm \Delta D_{e}$ (change in $D_{e}$ ) in the $\pm Y$ direction.

3: Compute positions of inner control points $\dot{P}_{K 1}^{e}, \hat{P}_{K 2}^{e}, \hat{P}_{K 1}^{m}, \hat{P}_{K 2}^{m}, \dot{P}_{S 5}^{e}$ and $\dot{P}_{S 6}^{e}$ using IA.

A similar approach is taken to change the depth $D_{m}$ at station-2 so that control point positions of the keel lines in both the middle and run regions, and the lower line of station-2 are modified. Similarly, the depth $D_{r}$ at station-3 is changed to $D_{r} \pm \Delta D_{r}$ by just modifying the control point positions of the keel line(s) in the run region and the lower line of station-3.

\subsubsection{Bow Angle Shape Operator}

The bow angle shape operator modifies the angle $\beta$ which is defined between positive $Z$ direction and bow line as shown in Figure 2 (b). Modification of the bow angle also affects the shape of the chine line(s) and keel line(s) in the entrance region. The approach taken to implement the bow angle shape operator is summed up by the following pseudo-code:

1: The control points $P_{C 3_{i: o}}^{e}, P_{K 3}^{e}, P_{D 0}^{e}$ and the upper point of the bow line are fixed.

2: Change the bow angle $\beta$ and position the lower point of the bow line without changing the vertical length $H$ of the bow line.

3: Make $P_{C 0_{i, o}}^{e}$ and $P_{K 0}^{e}$ to be the lower point of the bow line ( $F_{b}$ is kept constant).

4: Compute positions of inner control points $\dot{P}_{C 1_{i ; o}}^{e}, \hat{P}_{C 2_{i ; o}}^{e}, \hat{P}_{K 1}^{e}$ and $\dot{P}_{K 2}^{e}$ using IA. 


\subsubsection{Entrance Angle Shape Operator}

The entrance angle $\theta$ is defined between the first polygon segment of the deck line in the entrance region and Z-Y plane (see Figure 2 (b)). The entrance angle has significant influence on the hull shape in terms of its performance and aesthetics. The pseudo-code below summarizes the approach that is used for the implementation of this shape operator:

1: The control points $P_{D 0}^{e}$ and $P_{D 3}^{e}$ are fixed.

2: Move $P_{D 1}^{e}$ in the $\pm Z$ direction so that the desired angle $\theta$ is achieved.

3: Compute positions of inner control points $\dot{P}_{D 1}^{e}$ and $\dot{P}_{D 2}^{e}$ using IA.

\subsubsection{Sheer Angle Shape Operator}

The sheer angle shape operator modifies the sheer angle $\alpha$ between the deck plane and $Z-X$ plane (see Figure 7). This shape operator affects the shape of the deck line, bow line and upper lines of station- 1 and station- 2 . The pseudo-code for this shape operator is summarized as follows:

1: Create a plane (deck plane) coinciding with $P_{D 3}^{r}$ having angle of $\alpha$ with $Z-X$ plane ( see Figure 7).

2: Project the control point $P_{D 0}^{e}$ onto the deck plane in the direction of $\hat{a}$ while keeping $\beta$ constant.

3: Project the control points $P_{D 3}^{e}, P_{D 0}^{m}, P_{D 3}^{m}$ and $P_{D 0}^{r}$ onto the deck plane in the $\pm Y$ direction.

4: Make $P_{S 0}^{e}$ to be $P_{D 3}^{e}$ and $P_{S 0}^{m}$ to be $P_{D 3}^{m}$.

5: Compute positions of inner control points $\hat{P}_{D 1}^{e}, \hat{P}_{D 2}^{e}, \hat{P}_{D 1}^{m}, \hat{P}_{D 2}^{m}, \dot{P}_{D 1}^{r}, \dot{P}_{D 2}^{r}, \hat{P}_{S 1}^{e}, \dot{P}_{S 2}^{e}, \dot{P}_{S 1}^{m}$ and $\dot{P}_{S 2}^{m}$ using IA.

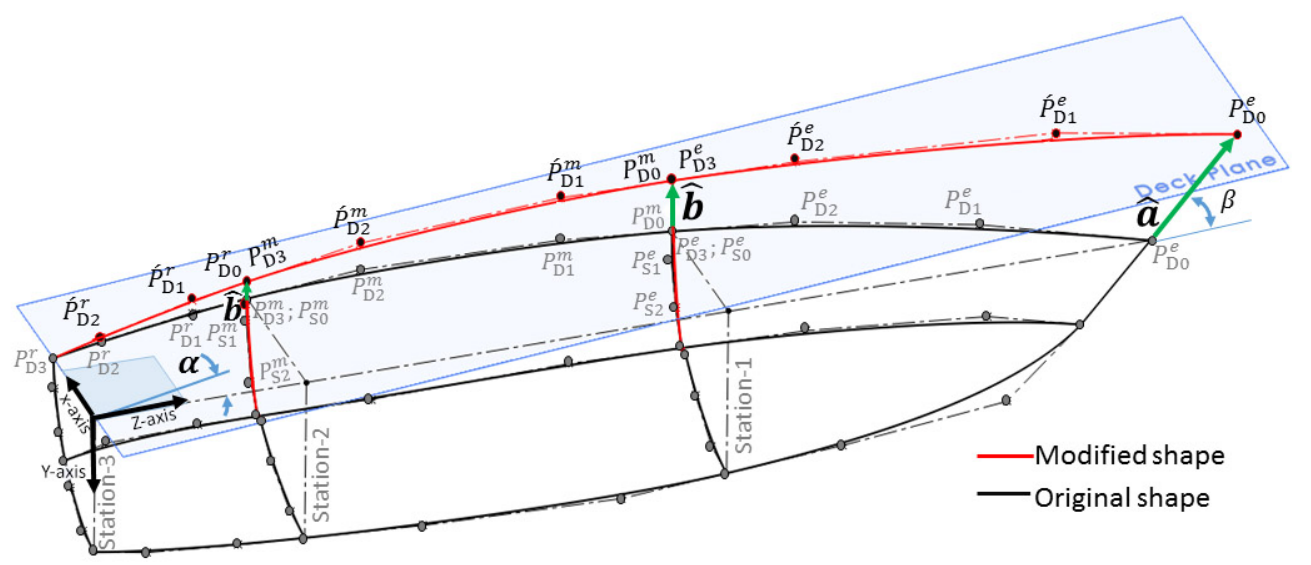

Figure 7. Shear angle modification.

\subsubsection{Bow Vertical Length Shape Operator}

The bow vertical length shape operator modifies the vertical length of the bow line (see Figure 8). The approach used to implement this shape operator is summed up by the following pseudo-code:

1: The control points $P_{D 0}^{e}, P_{C 0_{i, o}}^{e}$ and upper point of bow line are fixed.

2: Move the lower point of bow line by $\pm \Delta H$ in the positive or negative $\hat{d}$ direction so that the desired value for $H$ is achieved (Notice that the bow angle $\beta$ is kept constant, see Figure 8).

3: Make $P_{K 0}^{e}$ to be the lower point of the bow line.

4: Compute positions of inner control points $\hat{P}_{K 1}^{e}$ and $\dot{P}_{K 2}^{e}$ using IA.

\subsubsection{Chine Thickness Shape Operator}

Individual modification for the chine thickness parameters $C_{e}, C_{m}$ and $C_{r}$ is performed using this shape operator. $C_{e}$ is defined at station- 1 and its change affects the upper and lower lines of station-1, inner and outer chine lines in both entrance and middle regions. The approach used to modify $C_{e}$ is summed up by the pseudo-code below and similar approach can be used for the modification of $C_{m}$ and $C_{r}$. 


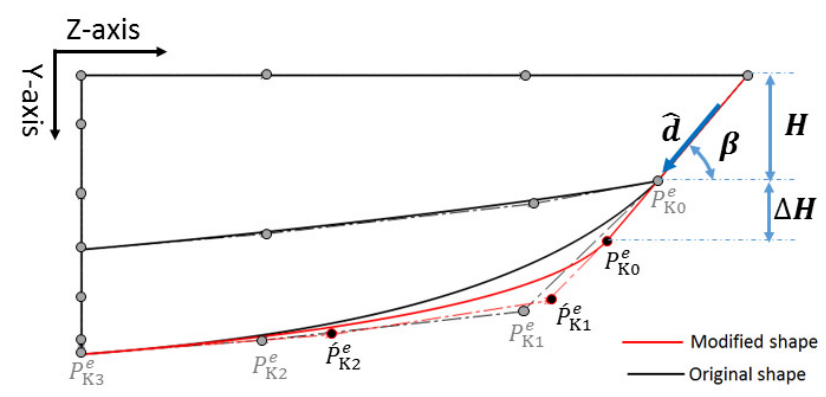

Figure 8. Modifying bow vertical length.

1: The control points $P_{S 0}^{e}, P_{S 7}^{e}, P_{C 0_{i}}^{e}, P_{C 0_{o}}^{e}, P_{C 3_{i}}^{m}$ and $P_{C 3_{o}}^{m}$ are fixed.

2: If a positive (negative) value for $\Delta C_{e}$ (change in $C_{e}$ ) is given as input.

3: $\quad$ Move $P_{C 3_{o}}^{e}$ and $P_{C 0_{o}}^{m}$ by $\Delta C_{e} / 2$ in the positive (negative) $X$ direction.

4: $\quad$ Move $P_{C 3_{i}}^{e^{3}}$ and $P_{C 0_{i}}^{m}$ by $\Delta C_{e} / 2$ in the negative (positive) $X$ direction.

5: end If

6: Make $P_{S 3}^{e}$ to be $P_{C 3_{o}}^{e}$ and $P_{S 4}^{e}$ to be $P_{C 3_{i}}^{e}$.

7: Compute positions of inner control points $\dot{P}_{C 1_{i: o}}^{e}, \dot{P}_{C 2_{i, o}}^{e}, \dot{P}_{C 1_{i ; o}}^{m}, \dot{P}_{C 2_{i ; o}}^{m}, \dot{P}_{S 1}^{e}, \dot{P}_{S 2}^{e}, \hat{P}_{S 5}^{e}$ and $\dot{P}_{S 6}^{e}$ using IA.

\subsubsection{Keel Thickness Shape Operator}

The keel thickness shape operator is mostly used for creating flat bottom hulls and modifies the value of $K$. $K$ is zero at the bow and increases along the negative $Z$ direction until station-1. It then remains constant until station-3 in this work. The following pseudo-code summarizes the technique used to implement this shape operator:

1: The control points $P_{S 4}^{e}, P_{S 4}^{m}$ and $P_{S 4}^{r}$ are fixed.

2: Move $P_{S 7}^{e}, P_{S 7}^{m}$ and $P_{S 7}^{r}$ by $\Delta K$ (change in $K$ ) in the $\pm X$ direction.

3: Move $P_{K 3_{o}}^{e}, P_{K 0_{o}}^{m}, P_{K 3_{o}}^{m}, P_{K 0_{o}}^{r}$ and $P_{K 3_{o}}^{r}$ by $\Delta K$ in the $\pm X$ direction $\left(P_{K 0_{o}}^{\delta}, P_{K 1_{o}}^{\delta}, P_{K 2_{o}}^{\delta}\right.$ and $P_{K 3_{o}}^{\delta}$ are the first, second, third and fourth control points of the offset keel line, respectively. $\delta$ represents the hull region and can be $e, m$ or $r)$.

4: Compute positions of inner control points $\dot{P}_{K 1_{o}}^{e}, \dot{P}_{K 2_{o}}^{e}, \dot{P}_{K 1_{o}}^{m}, \dot{P}_{K 2_{o}}^{m}, \dot{P}_{K 1_{o}}^{r}, \dot{P}_{K 2_{o}}^{r}, \dot{P}_{S 5}^{e}, \dot{P}_{S 6}^{e}, \dot{P}_{S 5}^{m}, \dot{P}_{S 6}^{m}, \dot{P}_{S 5}^{r}$ and $\dot{P}_{S 6}^{r}$ using IA.

\subsubsection{Chine Remover Shape Operator}

The chine remover shape operator transforms a double chine hull into a mono chine or no chine hull. Chine lines in three regions, and upper and lower lines of station-1, 2 and 3 are modified. The approach taken to implement this shape operator is summed up by the following pseudo-code:

1: Set the chine thicknesses $C_{e}, C_{m}$ and $C_{r}$ to zero.

2: Compute the middle point between the control points $P_{C 1_{i}}^{e}$ and $P_{C 1_{o}}^{e}$.

3: Make $P_{C 1_{i}}^{e}$ and $P_{C 1_{o}}^{e}$ to be the middle point.

4: Repeat line 2 and 3 for the control points $P_{C 2_{i, o}}^{e}, P_{C 3_{i ; o}}^{e}, P_{C 0_{i: o}}^{m}, P_{C 1_{i, o},}^{m}, P_{C 2_{i ; o}}^{m}, P_{C 3_{i ; o}}^{m}, P_{C 0_{i ; o}}^{r}, P_{C 1_{i ; o}}^{r}, P_{C 2_{i ; o}}^{r}$ and $P_{C 3_{i ; o}}^{r}$ (this makes the inner and outer chine lines be at the same position which is considered as single chine line).

5: Make $P_{S 3}^{e}$ and $P_{S 4}^{e}$ to be $P_{C 3_{i}}^{e}$.

6: Make $P_{S 3}^{m}$ and $P_{S 4}^{m}$ to be $P_{C 3_{i}}^{m}$.

7: Make $P_{S 3}^{r}$ and $P_{S 4}^{r}$ to be $P_{C 3_{i}}^{r}$.

8: Compute positions of inner control points $\dot{P}_{S 1}^{e}, \dot{P}_{S 2}^{e}, \dot{P}_{S 5}^{e}, \dot{P}_{S 6}^{e}, \dot{P}_{S 1}^{m}, \dot{P}_{S 2}^{m}, \dot{P}_{S 5}^{m}, \dot{P}_{S 6}^{m}, \dot{P}_{S 1}^{r}, \dot{P}_{S 2}^{r}, \dot{P}_{S 5}^{r}$ and $\dot{P}_{S 6}^{r}$ using IA.

In order to obtain the hull with no chine line(s), $G^{1}$ continuity is maintained by making the connecting tangents of upper and lower lines of station-1, 2 and 3 collinear. 


\subsubsection{Chine Inserter Shape Operator}

The chine inserter shape operator transforms a mono chine hull into double chine hull and operates on the chine thicknesses $C_{e}, C_{m}$ and $C_{r}$. The following approach is utilized to implement this shape operator:

1: Set $C_{e}, C_{m}$ and $C_{r}$.

2: The control points $P_{C 0_{i}}^{e}, P_{C 0_{o}}^{e}, P_{S 0}^{e}, P_{S 7}^{e}, P_{S 0}^{m}, P_{S 7}^{m}, P_{S 0}^{r}$ and $P_{S 7}^{r}$ are fixed.

3: Move $P_{C 3_{o}}^{e}$ by $C_{e} / 2$ in the positive $X$ direction and $P_{C 3_{i}}^{e}$ by $C_{e} / 2$ in the negative $X$ direction.

4: Move $P_{C 0_{o}}^{m}$ by $C_{e} / 2$ in the positive $X$ direction and $P_{C 0_{i}}^{m}$ by $C_{e} / 2$ in the negative $X$ direction.

5: Move $P_{C 3_{o}}^{m}$ by $C_{m} / 2$ in the positive $X$ direction and $P_{C 3_{i}}^{m}$ by $C_{m} / 2$ in the negative $X$ direction.

6: Move $P_{C 0_{o}}^{r}$ by $C_{m} / 2$ in the positive $X$ direction and $P_{C 0_{i}}^{r}$ by $C_{m} / 2$ in the negative $X$ direction.

7: Move $P_{C_{3} 3_{0}}^{r}$ by $C_{r} / 2$ in the positive $X$ direction and $P_{C 3_{i}}^{r}$ by $C_{r} / 2$ in the negative $X$ direction.

8: Make $P_{S 3}^{e}$ to be $P_{C 3_{o}}^{e}$ and $P_{S 4}^{e}$ to be $P_{C 3_{i}}^{e}$.

9: Make $P_{S 3}^{m}$ to be $P_{C 3_{o}}^{m}$ and $P_{S 4}^{m}$ to be $P_{C 3_{i}}^{m_{i}}$.

10: Make $P_{S 3}^{r}$ to be $P_{C 3_{o}}^{r}$ and $P_{S 4}^{r}$ to be $P_{C 3_{i}}^{r}$.

11: Compute positions of inner control points $\dot{P}_{S 1}^{e}, \dot{P}_{S 2}^{e}, \dot{P}_{S 5}^{e}, \dot{P}_{S 6}^{e}, \dot{P}_{S 1}^{m}, \dot{P}_{S 2}^{m}, P_{S 5}^{m}, \dot{P}_{S 6}^{m}, \dot{P}_{S 1}^{r}, \dot{P}_{S 2}^{r}, \dot{P}_{S 5}^{r}, \dot{P}_{S 6}^{r}, \dot{P}_{C 1_{i: o}}^{e}$, $\dot{P}_{C 2_{i, o}}^{e}, \dot{P}_{C 1_{i, o}}^{m}, \dot{P}_{C 2_{i, o}}^{m}, \dot{P}_{C 1_{i, o}}^{r}$ and $\dot{P}_{C 2_{i, o}}^{r}$ using IA.

\subsubsection{Chine Position Shape Operator}

The chine position shape operator modifies the design parameters $F_{b}, F_{e}, F_{m}$ and $F_{r}$ so that positions of chine line(s) at station-1, 2 and 3 and at the bow are changed. The following pseudo-code summarizes the approach taken for this shape operator.

1: The control points $P_{C 0_{i, 0},}^{e}, P_{C 3_{i, 0}}^{m}, P_{S 0}^{e}$ and $P_{S 7}^{e}$ are fixed.

2: Create an $X-Z$ plane at $P_{C 3_{i, o}}^{e}$ or $P_{C 0_{i, 0}}^{m}$.

3: Move the $X-Z$ plane by $\pm \Delta F_{e}$ (change in $F_{e}$ ) in the $\pm Y$ direction.

4: Get the intersection point between the station lines of station- 1 and the $X-Z$ plane.

5: Move $P_{C 3_{i, 0}}^{e}, P_{C 0_{i, o}}^{m}$ and $P_{S 3}^{e}$ to the intersection point.

6: Move the ordinate of $P_{S 4}^{e}$ to the intersection point (to keep the parameter $C_{e}$ constant).

7: Compute the angle $\omega_{1}$ between the line connecting $P_{C 2_{i, o}}^{e}$ and $P_{C 1_{i, o}}^{m}$ and $Y-X$ plane (see Figure 9 (a)).

8: Form another line at the modified $P_{C 3_{i \circ}}^{e}$ at an angle $\omega_{1}$ with $Y-X$ plane.

9: Project the control points $P_{C 2_{i, o}}^{e}$ and $P_{C 1_{i, o}}^{m}$ onto this line perpendicularly to make connecting polygon segments collinear for $G^{1}$ continuity.

10: Compute positions of inner control points $\dot{P}_{S 1}^{e}, \dot{P}_{S 2}^{e}, \dot{P}_{S 5}^{e}$ and $\dot{P}_{S 6}^{e}$ using IA.

A similar approach is taken to modify the design parameters $F_{m}$ and $F_{r}$. To change the position of chine line(s) at the bow, position of the $X-Z$ plane is changed to $F_{b} \pm \Delta F_{b}$ in $\pm Y$ direction and the control point $P_{C 0_{i, o}}^{e}$ is moved to the intersection point of this plane with the bow line.

\subsection{Implementation of Shape Operators in Group-2}

Specific techniques are utilized for each shape operators in this group, which will be explained in the following sub-sections. Although no separate technique is utilized to the achieve fairness criterion for feature curves while implementing these shape operators, the experiments in Sec. 5 reveal that the modified feature curves and the hull are still fair.

\subsubsection{Minimum Radius of Curvature Shape Operator}

The minimum radius of curvature shape operator is designed to modify minimum radius of curvature of feature curves. The minimum radius of curvature of a cubic curve has locally a monotone increasing behavior when inner control points of this curve start moving simultaneously towards each other from the neighboring boundary control points. Such motion of the inner control points makes the feature curve to reach a point where there exists an upper bounding value of minimum radius of curvature. After this upper bounding value, further motion of inner control points exhibits a monotonic decreasing behavior in the minimum radius of curvature value. Therefore, this shape operator modifies the shape of a feature curve for the minimum radius of curvature which is between zero and its upper bounding value. Below pseudo-code illustrates the approach used to implement this shape operator for $R_{e k}$ of the keel line: 

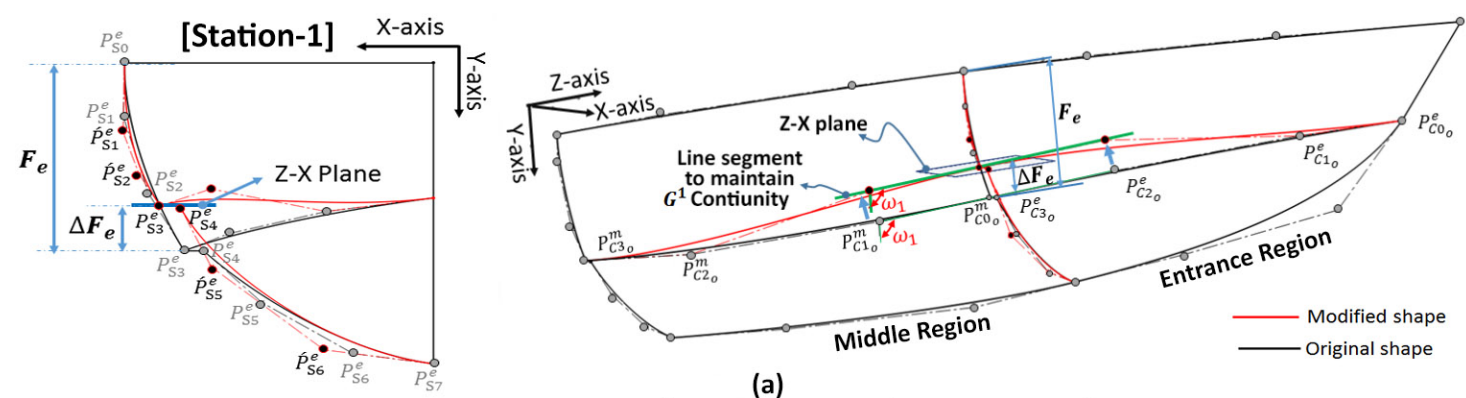

(a)

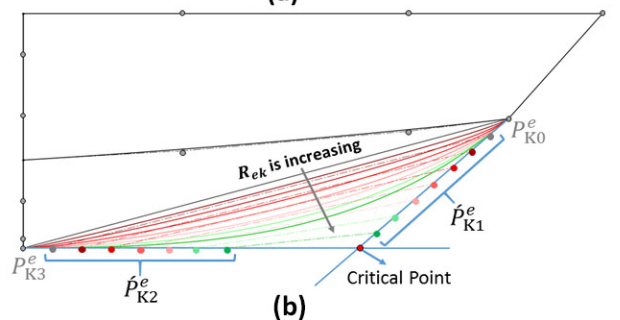

(b)

Figure 9. (a) Modification of the outer chine line at station-1 by using chine position shape operator. (b) Modification of the keel line in entrance region by using minimum radius of curvature shape operator.

1: The boundary control points $P_{K 0}^{e}$ and $P_{K 3}^{e}$ are fixed.

2: Move the inner control points $\hat{P}_{D 1}^{e}$ and $\hat{P}_{D 2}^{e}$ as done in the iterative approach in Sec. 4.2 until achieving the desired minimum radius of curvature for $R_{e k}$.

A similar approach is utilized to implement minimum radius of curvature shape operator for the design parameters $R_{e s 1}, R_{e s 2}, R_{m s 1}, R_{m s 2}, R_{r s 1}$ and $R_{r s 2}$. Figure 9 (b) shows the modification of minimum radius of curvature of the keel line in the entrance region.

\subsubsection{Station Concavity Shape Operator}

The concavity of station lines is the important aspect of the hull form aesthetics, which can be either concave up or concave down depending on the designer's choice (see Figure 10). The implementation of this shape operator on the lower line of station- 1 is summarized in the following approach:

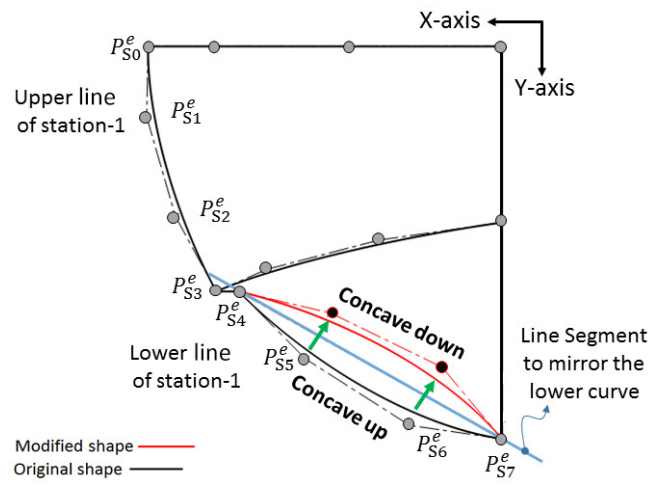

Figure 10. Applying station concavity shape operator on lower line of station-1.

1: The control points $P_{S 7}^{e}$ and $P_{S 4}^{e}$ are fixed.

2: Create a line segment between $P_{S 7}^{e}$ and $P_{S 4}^{e}$.

3: Mirror the inner control points $P_{S 5}^{e}$ and $P_{S 6}^{e}$ across this line segment (see Figure 10). 
Using this similar approach, the concavity of other station lines of station-1, 2 and 3 can be changed. To adjust the curve's concavity degree, the minimum radius of curvature shape operator should be applied to the feature curves with the station concavity shape operator.

\section{Results and Discussion}

For the implementation and verification of proposed shape operators, a semi-displacement yacht hull is generated by using the proposed design framework and taken as an input yacht hull. Numeric values for the design parameters of the hull can be seen in Table 4. Figure 11 shows the implementation results of the proposed shape operators that are applied on the entrance, middle and run regions of the hull. The first row in Figure 11 shows the input yacht hull models, while the modified hull models are shown in the next two rows. On the top, parameter values and their changes can be seen. For example, the length of the middle region $\left(L_{m}\right)$ is first increased by inputting the change in length $\left(\Delta L_{m}\right)$ as $3 m$, and later it is decreased by inputting the change in length $\left(\Delta L_{m}\right)$ as $-2 m$. According to these experiments, $G^{1}$ continuities are maintained between the feature curves of the neighboring regions of the hull, and hull fairness is ensured as well.

Table 4. Numeric values for the design parameters of the input hull.

\begin{tabular}{|l|l|l|l|l|l|l|l|}
\hline Parameters & Values & Parameters & Values & Parameters & Values & Parameters & Values \\
\hline$L_{e}$ & $8 \mathrm{~m}$ & $D_{m}$ & $3.21 \mathrm{~m}$ & $C_{m}$ & $0.15 \mathrm{~m}$ & $R_{r s 1}$ & $1.42 \mathrm{~m}$ \\
\hline$L_{m}$ & $8 \mathrm{~m}$ & $D_{r}$ & $2 \mathrm{~m}$ & $C_{r}$ & $0 \mathrm{~m}$ & $R_{r s 2}$ & $1.92 \mathrm{~m}$ \\
\hline$L_{r}$ & $4 \mathrm{~m}$ & $F_{e}$ & $2.5 \mathrm{~m}$ & $R_{e k}$ & $3.25 \mathrm{~m}$ & $H$ & $1.5 \mathrm{~m}$ \\
\hline$B_{e}$ & $6.4 \mathrm{~m}$ & $F_{m}$ & $2 \mathrm{~m}$ & $R_{e s 1}$ & $2.04 \mathrm{~m}$ & $K$ & $0 \mathrm{~m}$ \\
\hline$B_{m}$ & $6.32 \mathrm{~m}$ & $F_{r}$ & $1.5 \mathrm{~m}$ & $R_{e s 2}$ & $3.16 \mathrm{~m}$ & $\beta$ & $50^{\circ}$ \\
\hline$B_{r}$ & $4.5 \mathrm{~m}$ & $F_{b}$ & $1.3 \mathrm{~m}$ & $R_{m s 1}$ & $0.88 \mathrm{~m}$ & $\alpha$ & $0^{\circ}$ \\
\hline$D_{e}$ & $3.34 \mathrm{~m}$ & $C_{e}$ & $0.19 \mathrm{~m}$ & $R_{m s 2}$ & $3.01 \mathrm{~m}$ & $\theta$ & $30^{\circ}$ \\
\hline
\end{tabular}

Parameter tuning: In this study, the total number of curve divisions for curvature distribution $\xi$ is set to 1000 for all shape operators in Group-1. In other words, during each iteration the radius of curvature of a feature curve is calculated at 1000 equally spaced different points along the feature curve's parametric length. We set value of $\xi$ equal to 1000 , because with a higher value of $\xi$, a more accurate value of $F$ can be computed. The selection of the division constant $\mu$ is done based on the computational time taken for the IA and value of the objective function. For shape operators in Group-1, $\mu$ is taken as 15 . If the value of $\mu$ increases further, the change in the value of the objective function becomes negligibly small (as seen from Figure 12). Figure 12 shows plots for the objective function and $\mu$ in the case of deck line, keel line, inner and outer chine lines in the middle region when the elongation operator is applied. $\mu$ ranges from 3 to 50 . For the $\mu=15$ setting, $15 \times 15$ iterations are done to compute the inner control point positions. However, $\mu$ can be increased for more precise results which will result in a higher number of iterations and computation time. For the minimum radius of curvature shape operator in Group-2, $\mu$ and $\xi$ are taken as 350 and 10000 , respectively. It is confirmed that such setting results can produce better solutions.

Computational time: A PC having the Intel Core i7 6700, $3.4 \mathrm{GHz}$ processor and $16 \mathrm{~GB}$ memory is used for the experiments in this study. Table 5 shows the computational time to obtain the results shown in Figure 11. The time taken for the hull modification process is less than 4 seconds for all of the shape operators. Computation time heavily depends on the number of feature curves to be modified. The chine inserter shape operator has a higher computation time than all of the other shape operators in Group-1 because this shape operator has to optimize 12 feature curves including inner and outer chines in the entrance, middle and run regions, and upper and lower lines of station-1, 2 and 3. For shape operators in Group-2, the minimum radius of curvature shape operator has the maximum computation time due to a higher value setting of $\mu$ and $\xi$. The station concavity shape operator has the least computation time, because there is no feature curve that has to be optimized.

Verification of hull fairness: Experiments are conducted on the modified hulls to check hull fairness and $G^{1}$ continuities between connecting hull regions. Longitudinal and transverse zebra stripes are displayed on the hull 


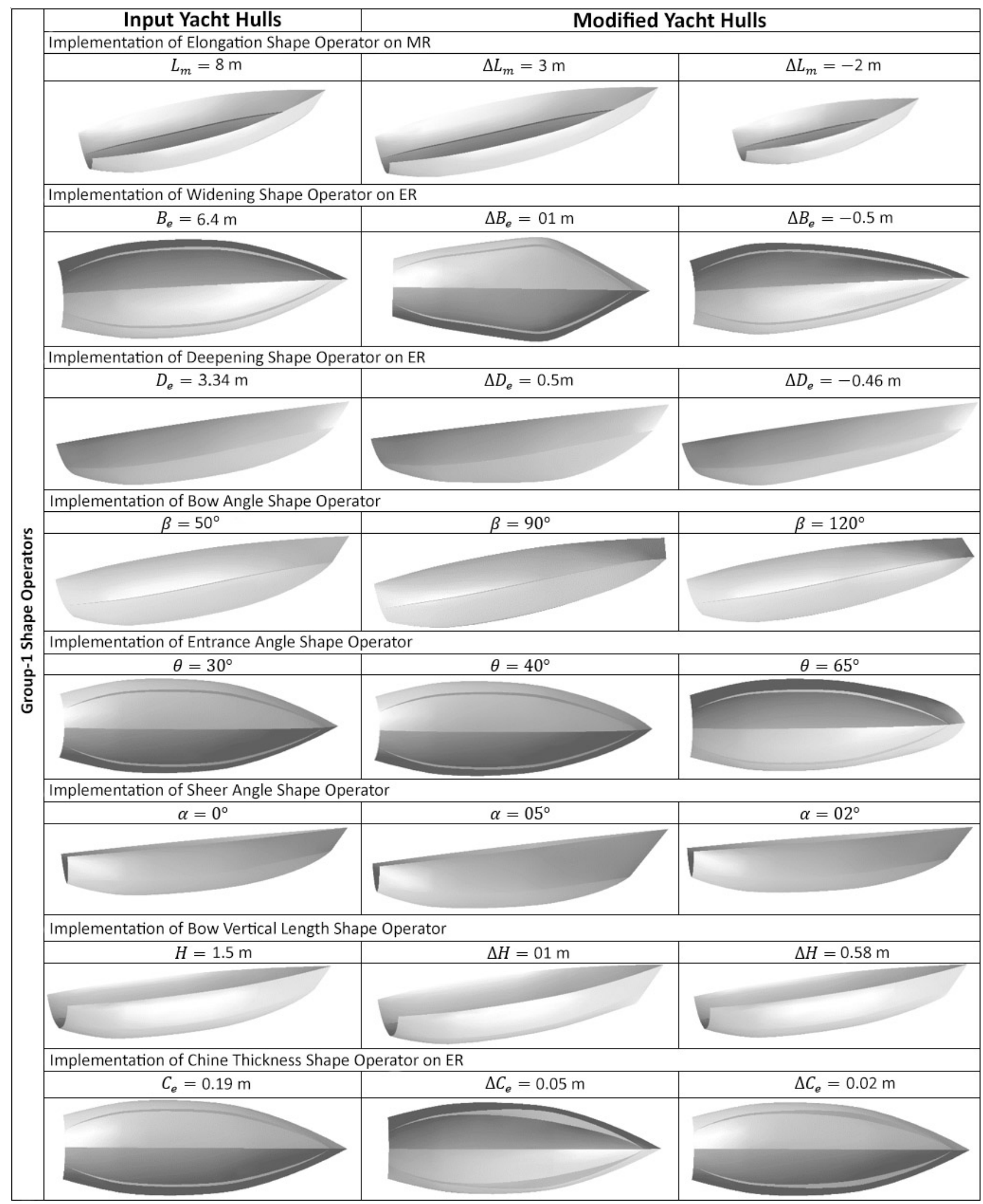




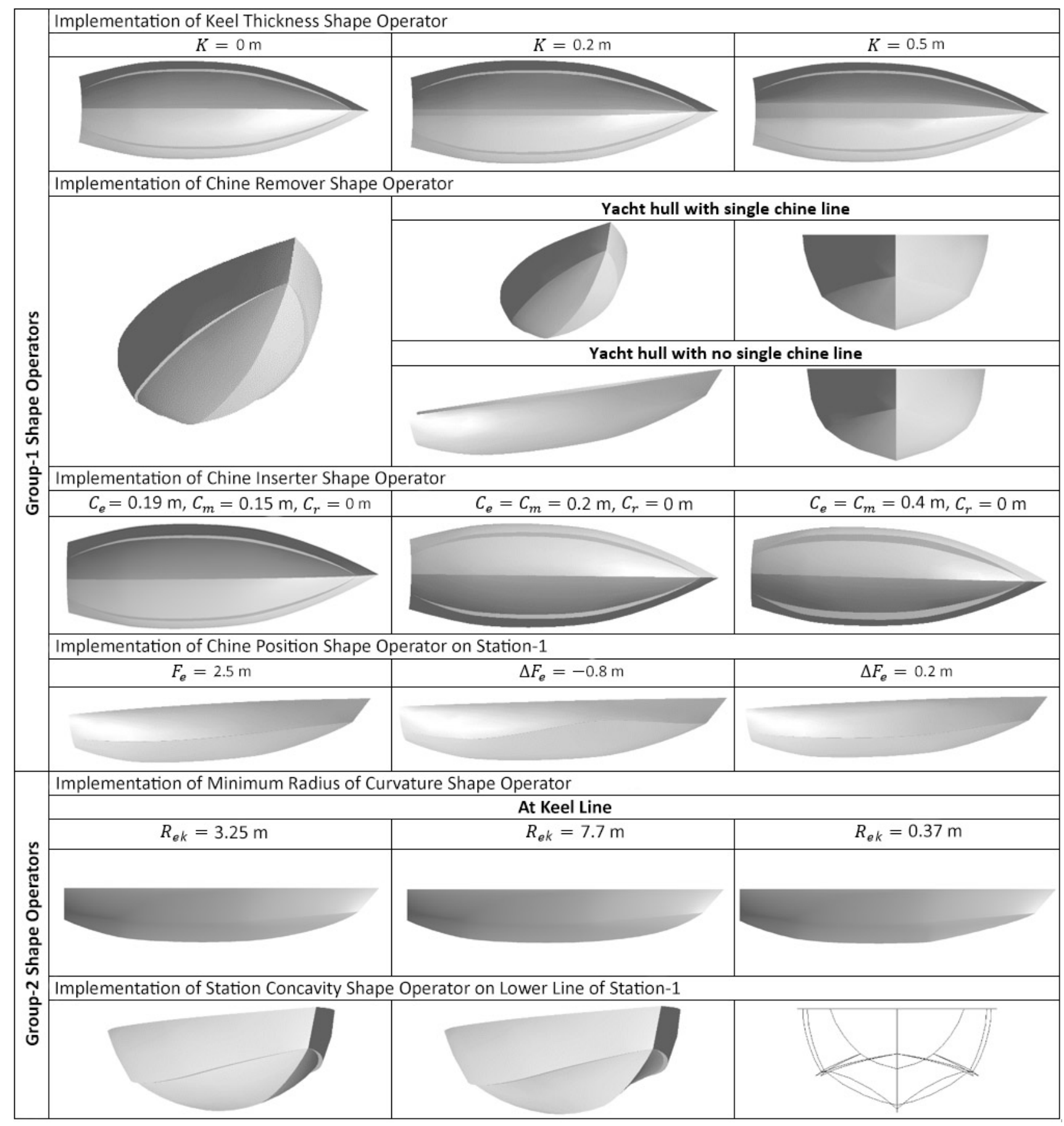

Figure 11. Application of shape operators and generated hulls.

surfaces that aid in visually inspecting the surface smoothness and geometric continuities between two connecting regions. In Figure 13 (a), irregular display of zebra stripes indicates the presence of bumps in the hull surfaces. As stated in Sec. 4.1, the presence of these bumps makes the hull unfair, thereby affecting its hydrodynamic behavior. While, in Figure 13 (b),(c),(d) and (e) smooth transition of the zebra stripes on the surfaces indicates smooth and fair hull surfaces. 

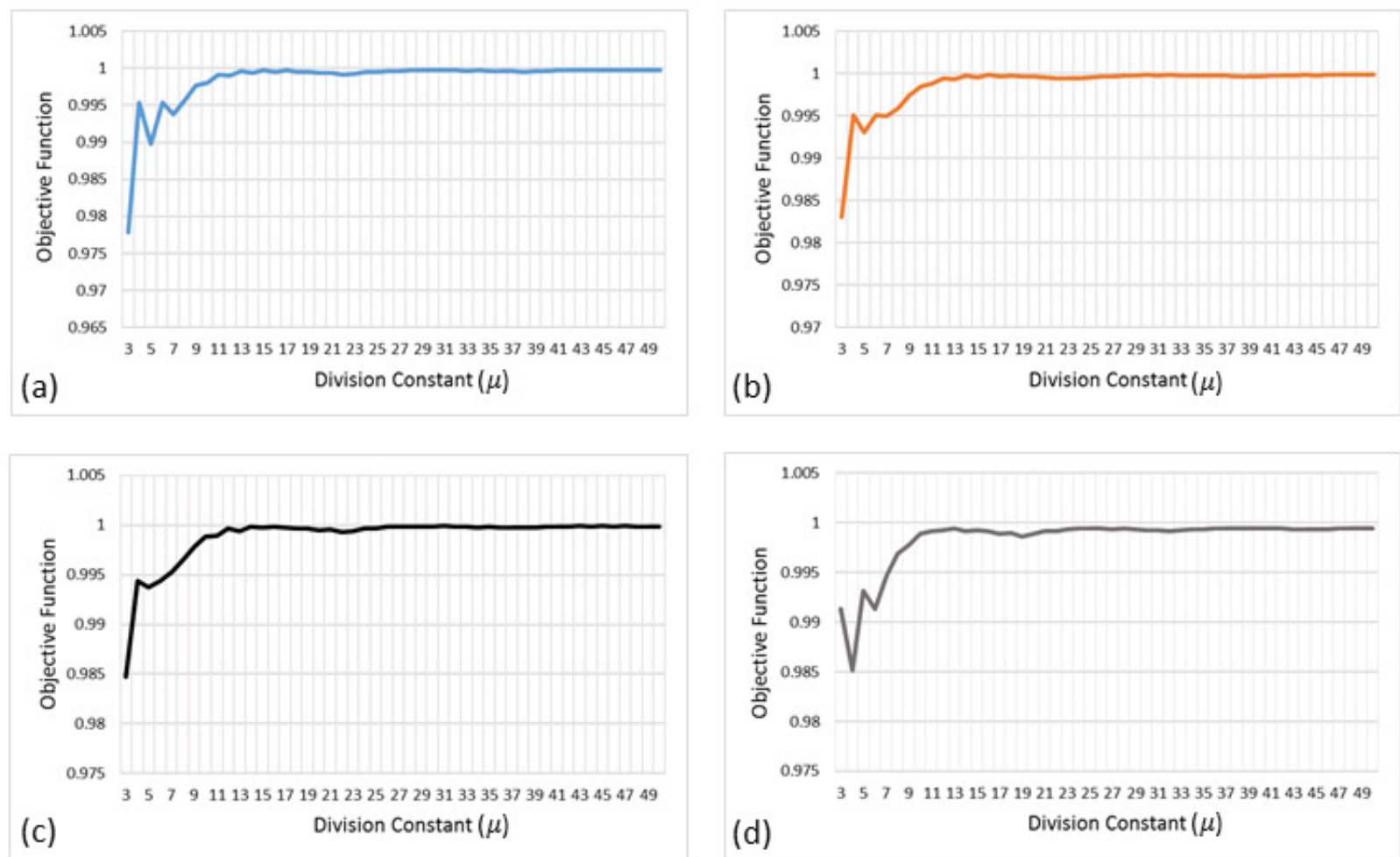

Figure 12. Change in the value of objective function $F$ with the increase of $\mu$ for: (a) Deck line (b) Inner chine line (c) Outer chine line (d) Keel line.

Table 5. Computational time for the hull modifications presented in Figure 11

\begin{tabular}{|c|c|c|c|c|c|c|c|c|}
\hline \multirow{2}{*}{\multicolumn{2}{|c|}{\begin{tabular}{|l} 
Shape Operator \\
Elongation Shape Operator on MR
\end{tabular}}} & \multirow{2}{*}{$\begin{array}{c}\begin{array}{c}\text { Design } \\
\text { Parameters }\end{array} \\
\Delta L_{m}\end{array}$} & \multirow{2}{*}{$\begin{array}{c}\xi \\
1000 \\
\end{array}$} & \multirow{2}{*}{$\begin{array}{c}\mu \\
15 \\
\end{array}$} & \multicolumn{2}{|c|}{$\begin{array}{l}\text { Parameter } \\
\text { Value } \\
\text { m (meter) }\end{array}$} & \multicolumn{2}{|c|}{$\begin{array}{c}\text { Computation } \\
\text { time } \\
\text { (seconds) }\end{array}$} \\
\hline & & & & & $3 m$ & $-2 m$ & 1.3 & 1.31 \\
\hline \multicolumn{2}{|c|}{ Widening Shape Operator of ER } & $\Delta B_{e}$ & 1000 & 15 & $1 \mathrm{~m}$ & $-5 m$ & 2.11 & 2.16 \\
\hline \multicolumn{2}{|c|}{ Deepening Shape Operator on ER } & $\Delta D_{e}$ & 1000 & 15 & $0.5 \mathrm{~m}$ & $-0.46 m$ & 0.969 & 0.931 \\
\hline \multicolumn{2}{|c|}{ Bow Angle Shape Operator } & $\beta$ & 1000 & 15 & $90^{\circ}$ & $120^{\circ}$ & 0.953 & 0.954 \\
\hline \multicolumn{2}{|c|}{ Entrance Angle Shape Operator } & $\theta$ & 1000 & 15 & $65^{\circ}$ & $40^{\circ}$ & 0.321 & 0.358 \\
\hline \multicolumn{2}{|c|}{ Sheer Angle Shape Operator } & $\alpha$ & 1000 & 15 & $05^{\circ}$ & $02^{\circ}$ & 1.64 & 1.58 \\
\hline \multicolumn{2}{|c|}{ Bow Vertical Length Shape Operator } & $\Delta H$ & 1000 & 15 & $0.1 \mathrm{~m}$ & $0.58 \mathrm{~m}$ & 0.23 & 0.174 \\
\hline \multicolumn{2}{|c|}{ Chine Thickness Shape Operator on ER } & $\Delta C_{e}$ & 1000 & 15 & $0.05 \mathrm{~m}$ & $0.02 \mathrm{~m}$ & 1.92 & 1.86 \\
\hline \multicolumn{2}{|c|}{ KeelThickness Shape Operator } & $K$ & 1000 & 15 & $0.5 \mathrm{~m}$ & $0.2 \mathrm{~m}$ & 0.953 & 0.906 \\
\hline \multicolumn{2}{|c|}{ Chine Position Shape Operator at Station-1 } & $\Delta F_{e}$ & 1000 & 15 & $-0.8 m$ & $0.2 \mathrm{~m}$ & 0.668 & 0.665 \\
\hline $\begin{array}{c}\text { Minimum Radius of } \\
\text { Curvature Shape Operator }\end{array}$ & Keel Line & $R_{e k}$ & 10000 & 350 & $7.7 \mathrm{~m}$ & $0.37 \mathrm{~m}$ & 3.27 & 2.36 \\
\hline \multirow{2}{*}{$\begin{array}{c}\text { Chine Remover Shape } \\
\text { Operator }\end{array}$} & \multicolumn{2}{|c|}{ Single chine line } & 1000 & 15 & & & \multicolumn{2}{|c|}{1.83} \\
\hline & \multicolumn{2}{|c|}{ No chine line } & 1000 & 15 & & & \multicolumn{2}{|c|}{2.09} \\
\hline \multicolumn{2}{|c|}{ Chine Inserter Shape Operator } & $C_{e}, C_{m}, C_{r}$ & 1000 & 15 & \multicolumn{2}{|c|}{$0.2 \mathrm{~m}, 0.2 \mathrm{~m}, 0 \mathrm{~m}$} & \multicolumn{2}{|c|}{3.82} \\
\hline \multicolumn{3}{|c|}{ Station Concavity Shape Operator on Lower Line of Station-1 } & & & & & \multicolumn{2}{|c|}{0.029} \\
\hline
\end{tabular}


The fairness of hull surfaces can also be inspected by plotting section curves. For a fair hull, section curves are smooth without any inflection points, which can be observed from Figure 13 (a) and (b). After inspecting zebra stripes and section curves, one can easily confirm that the hull shown in Figure 13 (a) is unfair and the hulls shown in Figures 13 (b),(c),(d) and (e) are fair. The results provided in Figures 13 (b),(c),(d) and (e) also show that zebra stripes line up smoothly at station- 1 and 2 so that $G^{1}$ continuities between the hull regions are achieved and the entire hull is fair.

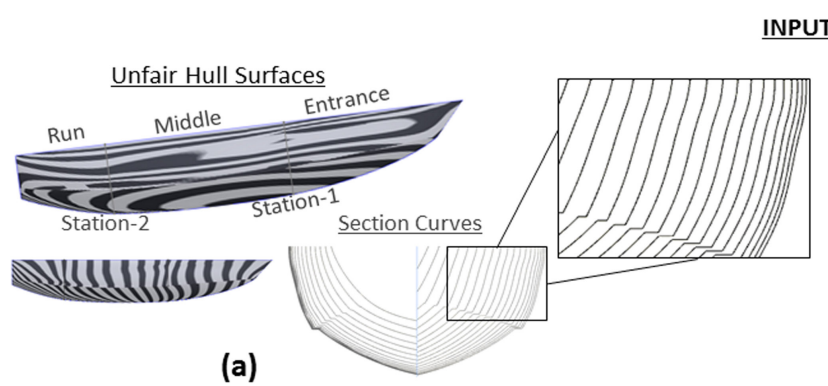

(a)

\section{PUT YACHT HULL}

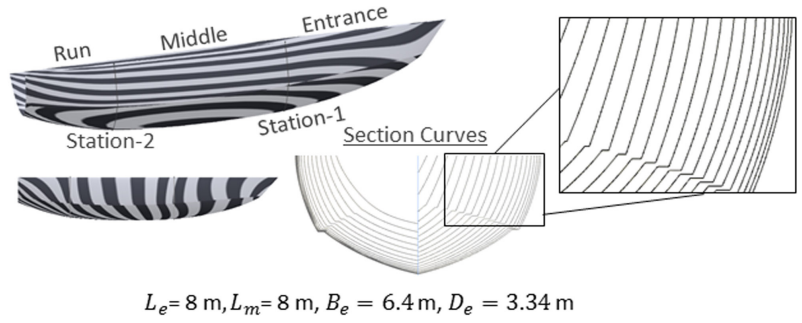

(b)

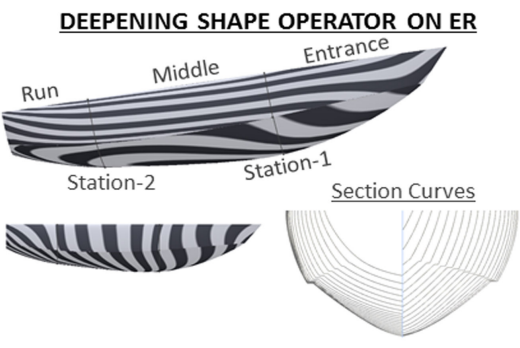

$L_{e}=8 \mathrm{~m}, L_{m}=8 \mathrm{~m}, B_{e}=6.4 \mathrm{~m}, D_{e}=3.84 \mathrm{~m}$

(c)

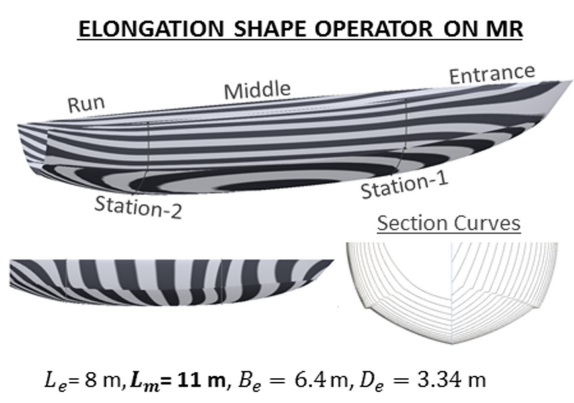

(d)

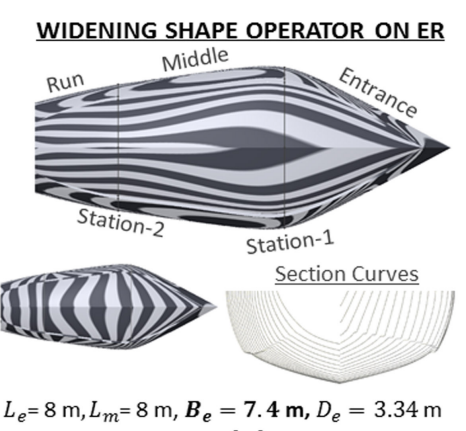

(e)

Figure 13. Inspection of hull fairness using longitudinal and transverse zebra stripes and section curves. (a) Unfair input yacht hull. (b) Fair input yacht hull. Fair yacht hull obtained after the application of: (c) Deepening (b) Elongation (d) Widening shape operator.

Similarity of curvature distributions after modification: Figure 14 shows curvature plots of the input and modified curves for the deck line, keel line, inner and outer chine lines in the middle region when the elongation shape operator is applied on the middle region for the design parameters $L_{m}=80$ and $\Delta L_{m}=30$. The objective function $F$ changes between 0 and 1 . Recall that curves are similar for the value of $F$ close to 1 . Therefore, Figure 14 indicates that modified feature curves are extensively similar to the input feature curves. It is also seen that the curvature plots change smoothly throughout the curve. This justifies that the modification of feature curves is carefully done and undesirable curve modification is eliminated. According to the results of the iterative approach, fairness of the feature curves is preserved.

Hydrostatic results: Table 6 presents the hydrostatic results and the form coefficients of the yacht hulls obtained after application of the elongation, widening, deepening, keel thickness and concavity shape operators on a given yacht hull.

Applying different shape operators: Different combinations of shape operators can be applied to an input hull in order to obtain completely different hull types or shapes. Figure 15 shows transformation of a semi-displacement yacht hull into a double chine shallow vee yacht hull and a rounded displacement yacht hull. The transformation of semi-displacement yacht hull into a double chine shallow vee yacht hull is achieved by applying the minimum radius of curvature shape operator on upper and lower lines of station-1, 2 and 3 first. The deepening shape operator on station-1, 2 and 3 (see Figure 15 (a)) is then applied. Likewise, the conversion of the semi-displacement yacht hull into a rounded displacement yacht hull is achieved by applying the chine remover and deepening shape operators on the entrance region first. The minimum radius of curvature operator on the lower line of station-1, 2 and 3 and the bow angle shape operator are then applied. Finally, the minimum radius of curvature shape operator on the keel line in the entrance region (see Figure 15 (b)) is utilized. The numeric values of design parameters for the modified hulls can be seen in Figure 15. 


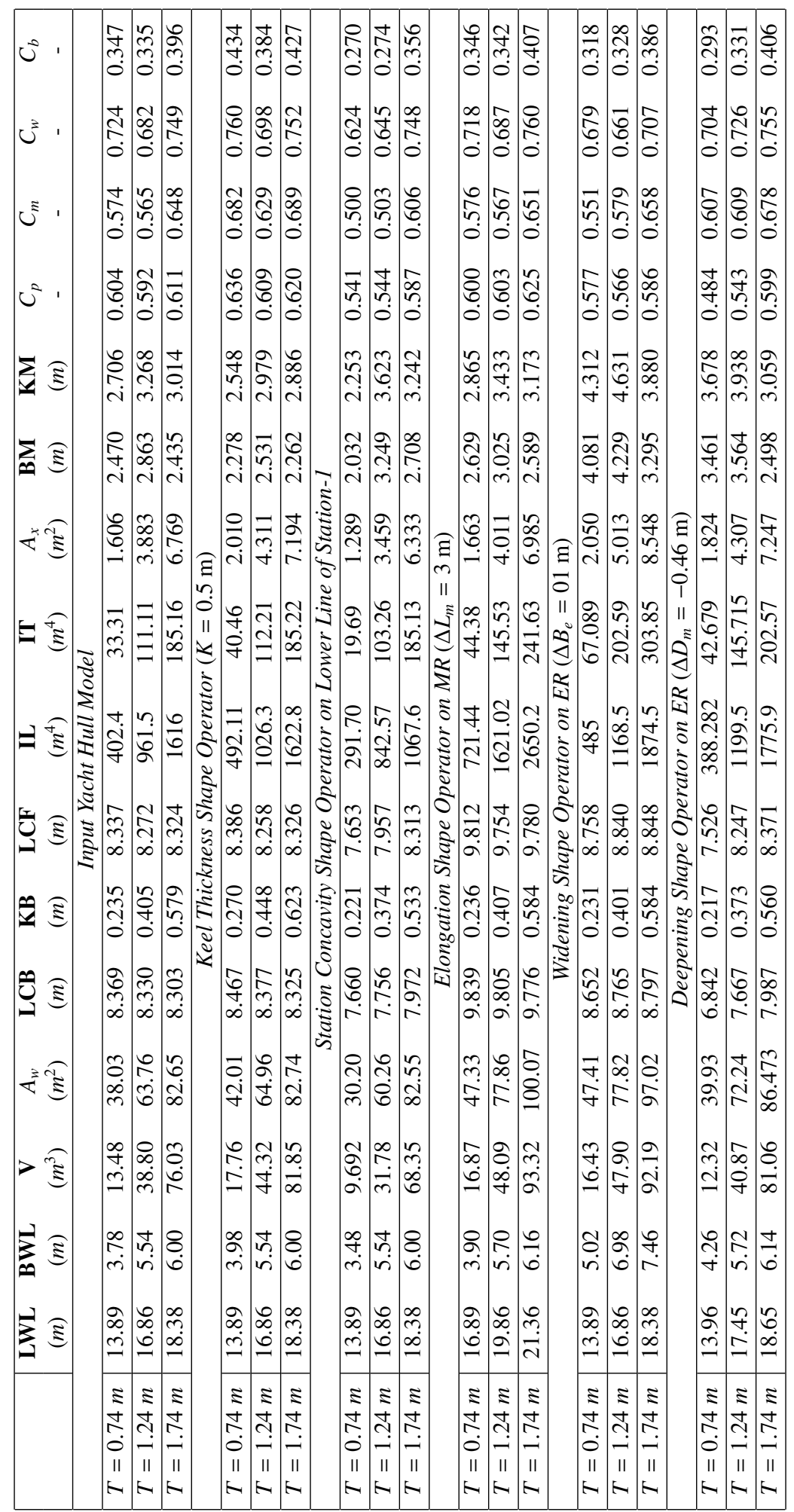



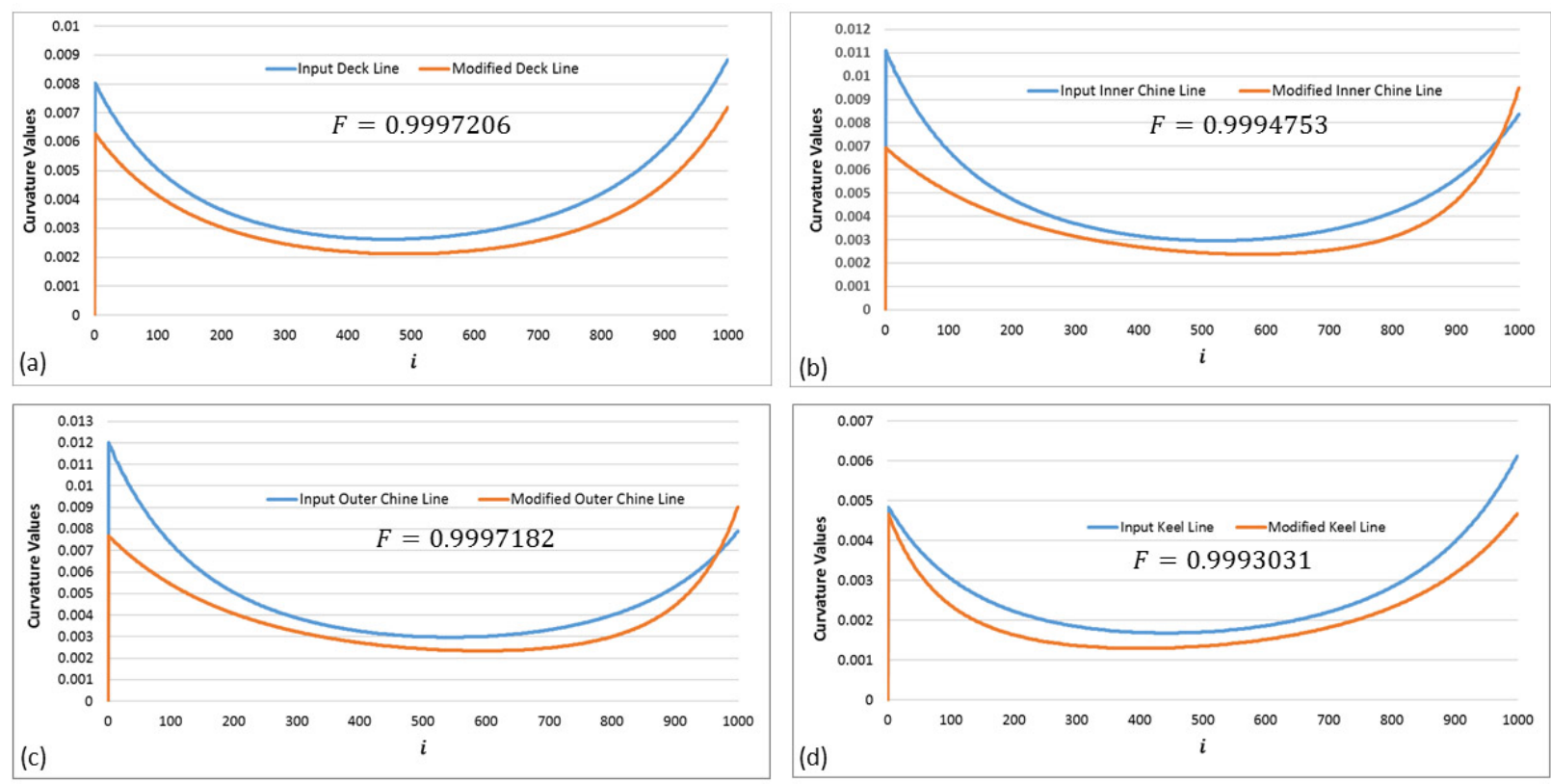

Figure 14. Curvature plots for the input and modified curves during the implementation of elongation shape operator on the middle region: (a) Deck line (b) Inner chine line (c) Outer chine line (d) Keel line.

\section{Semi-Displacement Hull}

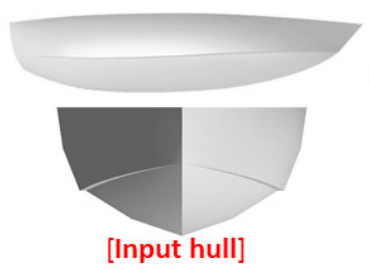

(1)

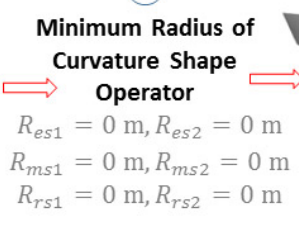

Minimum Radius of

$$
\Rightarrow \text { Operator }
$$

$=0 \mathrm{~m}, R_{\mathrm{ms} 2}=0 \mathrm{~m}$

$R_{r s 1}=0 \mathrm{~m}, R_{r s 2}=0 \mathrm{~m}$

(1)

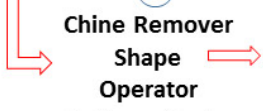

(with no chine)

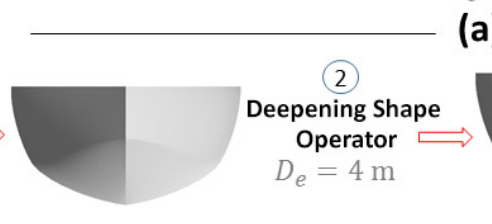

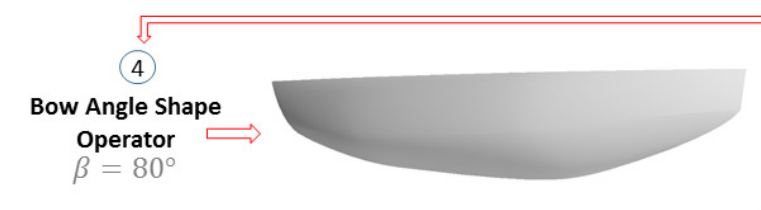

(b)

(2)

Double Chine Shallow Vee Hull

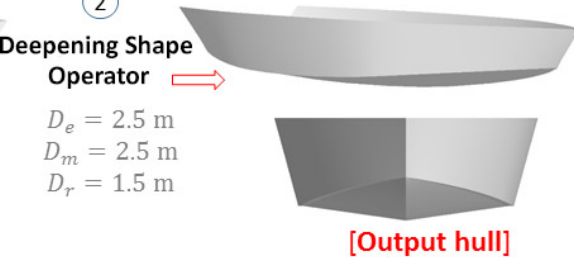

(3)

Minimum Radius of

Curvature Shape Operator

$R_{\text {es } 2}=3.8 \mathrm{~m}, R_{m s 2}=3.5 \mathrm{~m}$ $R_{r s 2}=2.2 \mathrm{~m}$

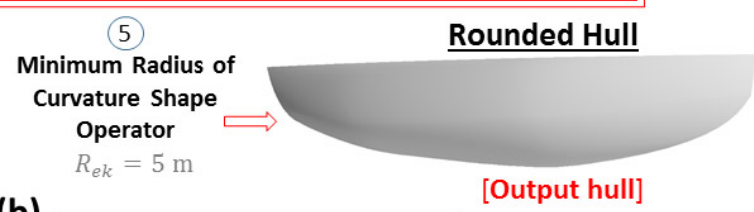

[Output hull]

Figure 15. Applying different shape operators consecutively.

We also apply a set of shape operators to obtain a region with a concave down at the start and concave up at the end. A yacht hull whose first Coons patch of the middle region consists of concave up upper lines of station-1 and station-2 is taken as input. The station concavity shape operator on the upper line of station- 1 and the elongation shape operator on middle region is applied. Figure 16 (b) shows the modified hull which is still fair when the smoothness of section curves of the hull is analyzed that is shown in Figure 16 (c).

Possible design scenario: Figure 17 shows hierarchical hull structure for the proposed design framework. There 


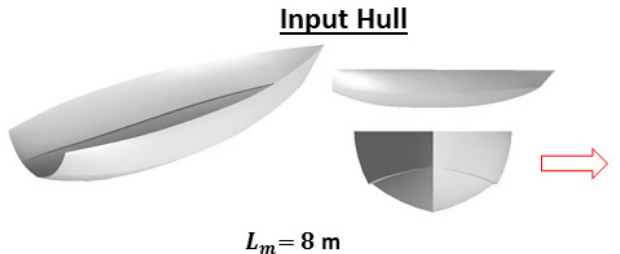

(a)

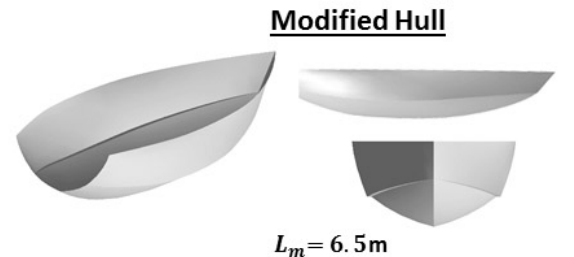

(b)

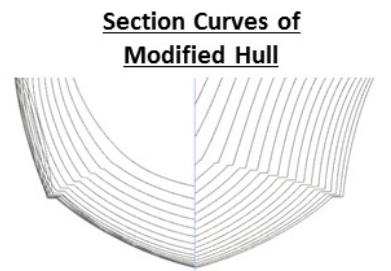

(c)

Figure 16. (a) Input semi-displacement yacht hull. (b) Modified yacht hull obtained after applying the elongation shape operator on the middle region and station concavity shape operator on the upper line of station-1. (c) Section curves' plot of the modified yacht hull.

are three parent hull types from which new hull variations can be inherited. A possible way to utilize the design framework is that all parent hulls with their design variations can be generated and stored in the hull database in advance. Designers then retrieve the hulls that they like and then modify them using the introduced shape modifiers. If they like to generate a different hull type for a given hull, a sequence of shape modifiers can also be used as illustrated in Figure 15.

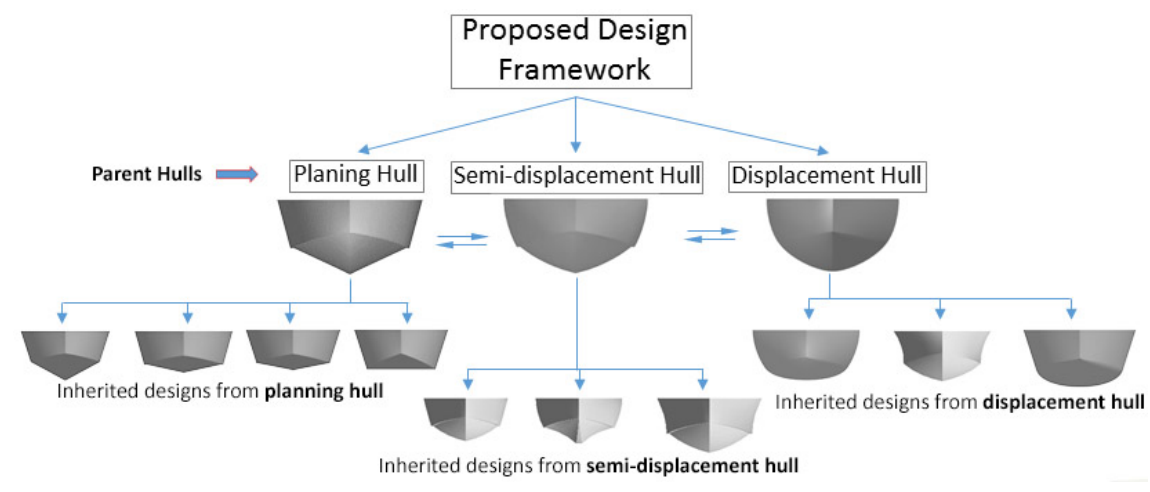

Figure 17. Hulls can be classified according to their hull types. Desired hull can be retrieved and used for the further modification.

\section{Conclusion and Future Works}

This paper has presented a novel design framework for the creation and parametric design modification of yacht hulls. Shape operators are developed for design modifications of a yacht hull and operate on the hull. Each shape operator is developed while taking three important quality criteria into account such as hull fairness and independent modification of the parameters. An objective function is introduced for the fair modification of feature curves. The experiments in this study showed that fair and feasible modification of input hulls can be achieved when the shape operators are applied. Furthermore, yacht hulls with higher design variations can be obtained with the help of shape operators.

In future work, the authors plan to extend this framework for the parametric design modification of multi-hull yachts (such as catamaran hulls) and hulls with three or more chines. An open source graphic interface will be created for users and will be published on the internet.

\section{Acknowledgments}

The authors would like to pay their deepest gratitude to The Scientific and Technological Research Council of Turkey (TUBITAK) for sponsoring this project (Project Number: 214M333). 


\section{References}

[1] D. E. Calkins, R. D. Schachter, L. T. Oliveira, An automated computational method for planing hull form definition in concept design, Ocean Engineering 28 (3) (2001) 297-327.

[2] F. Pérez-Arribas, Parametric generation of planing hulls, Ocean Engineering 81 (2014) 89-104.

[3] A. Mancuso, Parametric design of sailing hull shapes, Ocean Engineering 33 (2) (2006) 234-246.

[4] F. L. Pérez, J. A. Clemente, J. A. Suárez, J. M. González, Parametric generation, modeling, and fairing of simple hull lines with the use of non uniform rational b-spline surfaces, Journal of Ship Research 52 (1) (2008) 1-15.

[5] H. C. Kim, H. Nowacki, Parametric design of complex hull forms, Journal of Ship and Ocean Technology 9 (1) (2005) $47-63$.

[6] S. F. Zalek, R. F. Beck, M. G. Parsons, Nonlinear hullform transformation for use with design optimization, in: Proceedings of the 2008 Grand Challenges in Modeling and Simulation Conference, Simulation series, Vol. 40, 2008, pp. $227-238$.

[7] J. H. Nam, M. G. Parsons, A parametric approach for initial hull form modeling using nurbs representation, Journal of Ship Production 16 (2) (2000) 76-89.

[8] C. Abt, S. Bade, L. Birk, S. Harries, Parametric hull form design-a step towards one week ship design, in: 8th international symposium on practical design of ships and other floating structures, 2001, pp. 67-74.

[9] F. Pérez, J. A. Suárez, Quasi-developable b-spline surfaces in ship hull design, Computer Aided Design 39 (10) (2007) $853-862$.

[10] S. Harries, C. Abt, K. Hochkirch, Hydrodynamic modeling of sailing yachts, in: The 15th Chesapeake Sailing Yacht Symposium, Annapolis, 2001.

[11] K. Hochkirch, K. Röder, C. Abt, S. Harries, Advanced parametric yacht design, in: High performance yacht design conference, Auckland, Citeseer, 2002.

[12] A. Rodríguez, L. Fernández-Jambrina, Programmed design of ship forms, Computer Aided Design 44 (7) (2012) $687-696$.

[13] G. Kuiper, Preliminary design of ship lines by mathematical methods, Journal of Ship Research 14 (1) (1970) $52-66$.

[14] A. J. Keane, A computer based method for hull form concept design: applications to stability analyses, Royal Institution of Naval Architects Transactions 130 (1988) 61-75.

[15] F. Pérez-Arribas, J. A. Suárez-Suárez, L. Fernández-Jambrina, Automatic surface modelling of a ship hull, Computer Aided Design 38 (6) (2006) 584-594.

[16] P. ZHANG, D. X. ZHU, W. H. LENG, Parametric approach to design of hull forms, Journal of Hydrodynamics 20 (6) (2008) 804-810.

[17] M. Ghassabzadeh, H. Ghassemi, An innovative method for parametric design of planing tunnel vessel hull form, Ocean Engineering. 60 (2013) 14-27.

[18] M. G. Parsons, Applications of optimization in early stage ship design, Ship Science \& Technology 3 (5) (2009) 9-32.

[19] T. W. Lowe, J. Steel, Conceptual hull design using a genetic algorithm, Journal of Ship Research 47 (3) (2003) $222-236$.

[20] A. Papanikolaou, Holistic ship design optimization, Computer Aided Design 42 (11) (2010) 1028-1044.

[21] E. Jacquin, Q. Derbanne, D. Bellevre, S. Cordier, B. Alessandrini, Hull form optimization using a free surface ranse solver, in: 25th Symposium on Naval Hydrodynamics, St Johns, Newfoundland and Labrador, Canada, 2004.

[22] J. S. Kouh, S. W. Chau, Computer-aided geometric design and panel generation for hull forms based on rational cubic bezier curves, Computer aided geometric design 10 (6) (1993) 537-549.

[23] E. Narli, K. Sariöz, The automated fairing of ship hull lines using formal optimisation methods, Turkish Journal of Engineering and Environmental Sciences 28 (3) (2004) 157-166.

[24] G. Farin, Curves and surfaces for computer-aided geometric design: a practical guide, Elsevier, 2014.

[25] G. Farin, N. Sapidis, Curvature and the fairness of curves and surfaces, IEEE Computer Graphics and Applications 9 (2) (1989) $52-57$.

[26] G. Farin, Class a bezier curves, Computer Aided Geometric Design 23 (7) (2006) 573-581.

[27] E. Sariöz, An optimization approach for fairing of ship hull forms, Ocean Engineering 33 (16) (2006) 2105-2118.

[28] P. Salvi, T. Várady, Local fairing of freeform curves and surfaces, in: Proceedings of the Third Hungarian Graphics and Geometry Conference, 2005.

[29] T. Kuragano, K. Kasono, B-spline curve generation and modification based on specified radius of curvature, WSEAS Transactions on Information Science and Applications 5 (11) (2008) 1607-1617.

[30] T. Kuragano, K. Kasono, Curve shape modification and fairness evaluation for computer aided aesthetic design, in: Computational Intelligence for Modelling Control \& Automation, 2008 International Conference on, IEEE, 2008, pp. 82-87.

[31] T. Kuragano, A. Yamaguchi, Nurbs curve shape modification and fairness evaluation for computer aided aesthetic design, in: Proceedings of the 7th WSEAS International Conference on Signal Processing, Computational Geometry \& Artificial Vision, Citeseer, 2007 , pp. 38-44. 